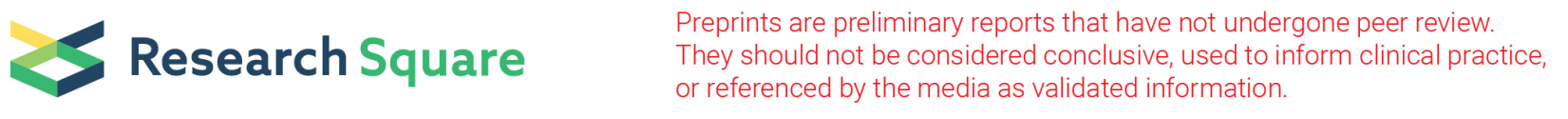

\title{
Case Control Association Study of WNT Signaling Pathway-related Genes and Osteoporosis Risk Among Chinese Postmenopausal Women
}

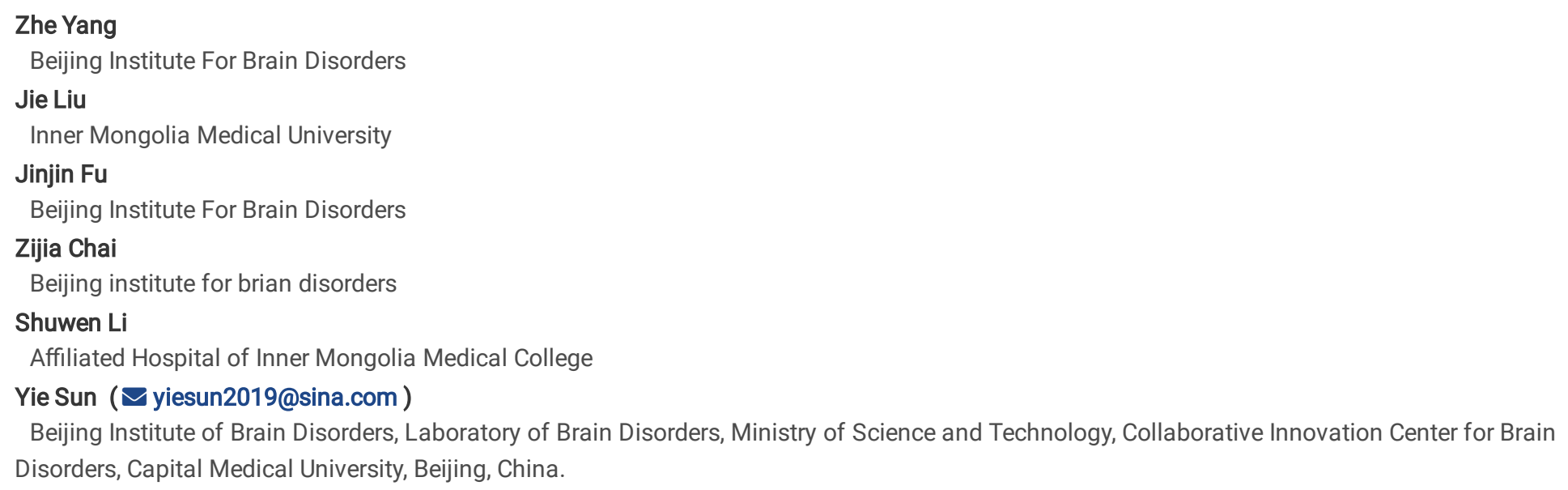

Keywords: Osteoporosis, WLS, WNT16, LRP5, Single nucleotide polymorphism, Multifactor dimensionality reduction

Posted Date: August 24th, 2020

DOI: https://doi.org/10.21203/rs.3.rs-62309/v1

License: (c) (i) This work is licensed under a Creative Commons Attribution 4.0 International License. Read Full License 


\section{Abstract}

Background: The WNT signaling pathway is involved in the regulation of bone homeostasis, and the effects of WNT signaling pathway related genes (WLS, WNT16 and LRP5) on osteoporosis risk among Chinese postmenopausal women is still unknown. Hence, we performed a casecontrol study to assess the association of WNT signaling pathway related genes and osteoporosis risk.

Methods: This study involved 1026 women (515 osteoporosis patients and 511 controls) of postmenopausal age who were randomly sampled from Xi'an 630 Hospital, Shaanxi Province, China. Eleven genetic polymorphisms in WLS (rs2566755, rs12407028, rs2566752 and rs7554551), WNT16 (rs3779381, rs3801387, rs917727 and rs7776725) and LRP5(rs2291467, rs11228240 and rs12272917) were selected and genotyped using the Agena MassARRAY iPLEX system. The association of the genetic polymorphisms and osteoporosis risk was assessed by odds ratios and $95 \%$ confidence intervals. The Multifactor Dimensionality Reduction (MDR) method was conducted to analyze SNP-SNP interaction.

Results: We found that LRP5 polymorphisms (rs2291467, rs11228240 and rs12272917) were significantly associated with a decreased risk of osteoporosis in homozygotes, both in recessive and additive models $(P<0.05)$. Stratification analysis showed that $L R P 5$ polymorphisms (rs2291467, rs11228240 and rs12272917) significantly decreased the osteoporosis risk in the subgroup of BMI $\leq 24(P<0.05)$ and that individuals carrying a heterozygote genotype of WNT16 polymorphisms (rs3779381, rs3801387, rs917727 and rs7776725) had a higher osteoporosis risk in the subgroup of $\mathrm{BMI}>24(P<0.05)$. We observed that three blocks (block 1: rs2566755 and rs12407028, block 2: rs3779381, rs3801387, rs917727 and rs7776725, block 3: rs2291467 and rs11228240), $\mathrm{T}_{\mathrm{rs} 2291467} \mathrm{~T}_{\mathrm{rs} 11228240}$ and $\mathrm{C}_{\mathrm{rs} 2291467} \mathrm{C}_{\mathrm{rs} 11228240}$ had a strong association with a lower risk of osteoporosis. Additionally, MDR analysis revealed that a four-locus model (rs2566752 and rs2566755 in WLS, rs7776725 in WNT16, rs12272917 in LRP5) was significantly associated with osteoporosis risk.

Conclusions: Our findings suggested that WLS, WNT16 and LRP5 genetic polymorphisms were associated with osteoporosis risk among Chinese postmenopausal women.

\section{Introduction}

Osteoporosis is a skeletal disorder characterized by low bone mineral density (BMD) and a compromised bone microarchitecture, which lead to increased risks of bone fragility and fracture[1]. Osteoporosis has become a major healthcare problem worldwide, affecting more than 200 million people[2]. There are gender differences in the occurrence of osteoporosis; postmenopausal women are more likely to have osteoporosis due to the sharp decline of estrogen production[3]. In China, the overall prevalence of osteoporosis ranges from $6.6-19.3 \%$ and increases with age[4]. It is estimated that the osteoporosis prevalence of Chinese women aged 50 years or older is more than $40 \%[4]$. The causes of osteoporosis are multifactorial, including age, sex, hormones, physical activity, diets, genetic factors and environmental factors. Among these, genetic factors play an important role in osteoporosis development. Moreover, increasing findings suggest that genes related to the WNT signaling pathway have effects on the onset and development of osteoporosis[5-9].

Wntless (WLS) is the key component required for WNT sorting and secretion and is also known as EVI or GPR177. WLS encodes a transmembrane protein that is required for the proper shuttling of WNT ligands involved in specified secretory pathways[10-12]. Overexpression of WLSleads to an interaction with WNT3A and VPS35, which suggests a nonredundant role of WLS on WNT regulation[13]. Fu et al. reported that disruption of WLS impairs the development of the body axis in mice[14]. In humans, Augustin et al. found that WLS is overexpressed in malignant astrocytoma and is related to the proliferation, survival and migration of glioma cells. Meanwhile, the expression level of WLS plays an essential role in some diseases, such as breast cancer, ovarian and gastric cancer[15-18]. Moreover, WLS has been identified as a novel locus for BMD and osteoporosis[19]. However, knowledge of the role of WLS in osteoporosis risk is limited to ethnicity, and the impact of the WLS pathway on the regulation of osteoporosis is unknown.

Wnt family member 16 (WNT16) spans $16 \mathrm{~kb}$ from the initiation to termination codons and encodes two protein isoforms (WNT16A and WNT16B) [20]. WNT16A is expressed in the pancreas, whereas WNT16B is highly expressed in the kidney, placenta, brain, heart and spleen[21]. WNT16 belongs to the WNT family, which regulates bone and mineral homeostasis[22]. The disruption of WNT16 in mice leads to lower cortical bone density and strength and an increased risk of fracture[23]. In premenopausal women, WNT16 polymorphisms are associated with peak BMD[24]. Recently, several genome-wide association studies (GWAS) have revealed that WNT16 polymorphisms are associated with BMD and fracture risk across multiple populations[24-27]. These studies suggest that WNT16 is vital for bone mass regulation, which might be a promising biomarker of osteoporosis.

Low-density lipoprotein receptor-related protein $5(L R P 5)$ is located on chromosome 11q13.4 and encodes a WNT receptor that regulates osteoblastic activity and bone mass[28]. LRP5 is also related to glucose and cholesterol metabolism, and thus, LRP5 is involved in the development of metabolic diseases, such as diabetes, obesity and vascular diseases[29]. Moreover, numerous studies have indicated that the $\angle R P 5$ gene influences $B M D$ and fracture[30]. Mouse models show that $L R P 5$ regulates bone formation by controlling osteoblast proliferation[31]. In humans, loss of $L R P 5$ expression is linked to osteoporosis-pseudoglioma syndrome (low BMD and skeletal fragility)[28]. However, there are no 
studies on the association of LRP5 polymorphisms (rs2291467, rs11228240 and rs12272917) with osteoporosis among Chinese postmenopausal women.

In this study, we enrolled 1026 individuals from Xi'an 630 Hospital and investigated the association between related genes of the WNT signaling pathway (WLS. rs2566755, rs12407028, rs2566752 and rs7554551; WNT16: rs3779381, rs3801387, rs917727 and rs7776725; LRP5: rs2291467, rs11228240 and rs12272917) and osteoporosis risk among Chinese postmenopausal women.

\section{Materials And Methods}

Study population

This case-control study was conducted in 515 osteoporosis patients (women) and 511 healthy postmenopausal women who were recruited from Xi'an 630 Hospital. All cases were defined by a bone mineral density (BMD) T score of less than - 2.5 in the lumbar spine (L1 to L4). The exclusion criteria included (1) patients with secondary osteoporosis, (2) patients treated with medications that may influence BMD, (3) postmenopausal individuals who were not on medication, and (4) individuals with other diseases, such as immune disorders, cancer, or metabolic or endocrine diseases. The controls were generally healthy subjects aged approximately 63 years. We collected basic and clinical characteristics from their medical records. This study was performed in accordance with the World Medical Association Declaration of Helsinki and was approved by the Ethics Committee of Xi'an 630 Hospital. Written informed consent was obtained prior to the study.

Genotyping

Four WLSSNPs (rs2566755, rs12407028, rs2566752 and rs7554551), four WNT16 SNPs (rs3779381, rs3801387, rs917727 and rs7776725) and three LRP5 SNPs (rs2291467, rs11228240 and rs12272917) were selected in this study based on data from the dbSNP database (https://www.ncbi.nlm.nih.gov/snp/) and 1000 Genomes Project (http://www.internationalgenome.org/). Genomic DNA was isolated from whole blood using a DNA extraction kit (GoldMag Co. Ltd., Xi囚an, China), and the DNA concentration was measured by a Nanodrop 2000 (Thermo Scientific, Waltham, Massachusetts, USA). Genotyping was carried out using the MassARRAY iPLEX system (Agena Bioscience, San Diego, CA, USA). The primers for this study were designed using the Agena MassARRAY Assay Design 3.0 Software (San Diego, California, USA, Supplemental Table 1). We managed and analyzed the data on the Agena Typer 4.0 Software (San Diego, CA, USA). Additionally, we used Haploview software (version 4.2) to perform haplotype analysis.

\section{Statistical analysis}

We performed statistical analysis using SPSS version 21.0 software (SPSS, Chicago, IL, USA). The $\chi 2$ test or $t$ test was applied to examine the distribution difference of characteristics. Deviation from Hardy-Weinberg equilibrium (HWE) of each SNP in the controls was tested using Fisher's exact test. The effects of genetic polymorphisms on osteoporosis risk, adjusted with or without age, were assessed by odds ratios (OR) and $95 \%$ confidential intervals $(95 \% \mathrm{Cl})$ using logistic regression. We also conducted haplotype analysis of the association of genetic polymorphisms with osteoporosis risk and calculated linkage disequilibrium (LD) using Haploview software (version 4.2) and PLINK software. Moreover, we used multifactor dimensionality reduction (MDR, version 3.0.2) to evaluate the effects of SNP-SNP interactions on osteoporosis risk. All tests were twosided, and the significance threshold set at $P<0.05$.

\section{Results}

Characteristics of the study population

The characteristics of all the participants are shown in Table 1, including age, body mass index (BMI), uric acid (UA), urea, fasting glucose, white blood cell (WBC), red blood cell (RBC), platelets (PLT), platelet distribution width (PDW), mean platelet volume (MPV), plateletocrit (PCT), L1, L2, L3 and L4. The mean ages of the cases and controls were $63.72 \pm 5.58$ and $62.87 \pm 4.68$ years, respectively. There were significant differences in age, UA, urea, fasting glucose, WBC, RBC, PLT, PDW, MPV and PCT between the two groups $(P<0.05)$. 
Table 1

Characteristics of the study population

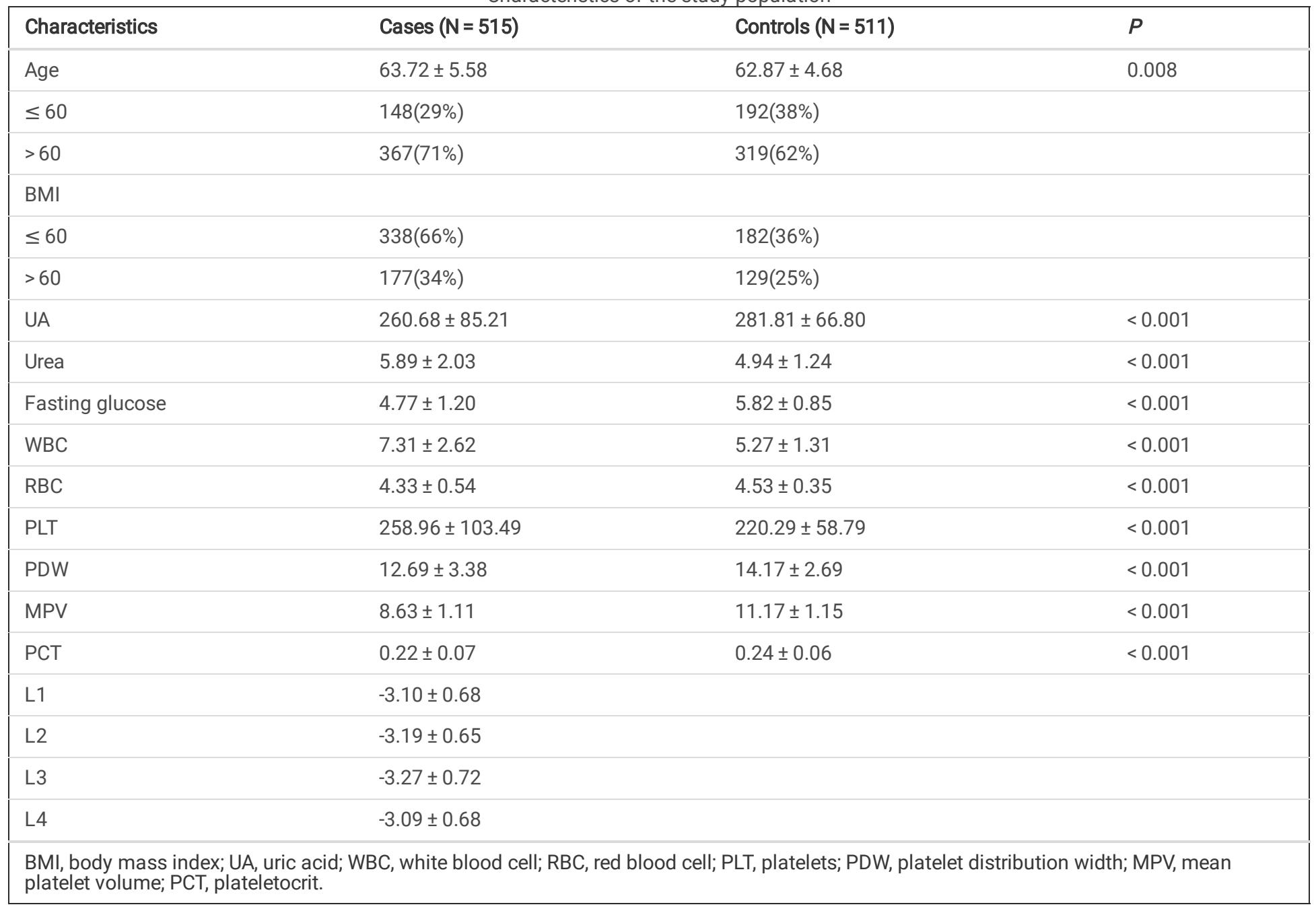

Association of genetic polymorphisms with osteoporosis risk

Basic information about the selected SNPs is presented in Supplemental Table 2. All the genetic polymorphisms were in Hardy-Weinberg equilibrium $(P>0.05)$. In the allele model, LRP5 polymorphisms (rs2291467, rs11228240 and rs12272917) were significantly associated with a decreased risk of osteoporosis (rs2291467: $\mathrm{OR}=0.77,95 \% \mathrm{Cl}=0.62-0.96, P=0.019 ; \mathrm{rs} 11228240: 0 \mathrm{R}=0.78,95 \% \mathrm{Cl}=0.63-0.96, P=0.022$; rs12272917: $\mathrm{OR}=0.76,95 \% \mathrm{Cl}=0.61-0.94, P=0.012)$.

As predicted by HaploReg, the functions of the genetic polymorphisms were related to promoter histone marks, enhancer histone marks, motif changes, NHGRI/EBI GWAS hits, GRASP QTL hits, selected eQTL hits, SiPhy cons, DNAse and proteins bound. In Table 2, we assessed the association of the genetic polymorphisms with osteoporosis risk in different inheritance models (homozygote, heterozygote, dominant, recessive and additive). After adjustment, individuals with the rs2291467 TT genotype had a lower risk of osteoporosis in homozygote $(\mathrm{OR}=0.17,95 \% \mathrm{Cl}=$ $0.07-0.45, P<0.001)$ and recessive models $(\mathrm{OR}=0.17,95 \% \mathrm{Cl}=0.07-0.45, P<0.001)$. In the additive model, a strong relationship was also observed between $\angle R P 5$ rs2291467 and osteoporosis risk $(\mathrm{OR}=0.77,95 \% \mathrm{Cl}=0.61-0.96, P=0.021)$. Meanwhile, rs 11228240 and rs 12272917 of $\angle R P 5$ were significantly related to decreased osteoporosis susceptibility in homozygote $(\mathrm{rs} 11228240: 0 \mathrm{R}=0.17,95 \% \mathrm{Cl}=0.07-0.45, P<0.001$; rs12272917: $\mathrm{OR}=0.23,95 \% \mathrm{Cl}=0.10-0.54, P=0.001)$, recessive $(\mathrm{rs} 11228240: \mathrm{OR}=0.17,95 \% \mathrm{Cl}=0.07-0.46, P<0.001 ; \mathrm{rs} 12272917: 0 \mathrm{R}=0.24$, $95 \% \mathrm{Cl}=0.10-0.55, P=0.001)$ and additive (rs11228240: $\mathrm{OR}=0.77,95 \% \mathrm{Cl}=0.62-0.97, P=0.025 ; \mathrm{rs} 12272917: 0 \mathrm{R}=0.76,95 \% \mathrm{Cl}=0.60-0.94, P=$ $0.013)$ models. 
Table 2

Association of genetic polymorphism with osteoporosis risk

\begin{tabular}{|c|c|c|c|c|c|c|c|}
\hline \multirow[t]{2}{*}{ Gene } & \multirow[t]{2}{*}{ SNP } & \multirow[t]{2}{*}{ Model } & \multirow[t]{2}{*}{ Genotype } & \multicolumn{2}{|l|}{ With adjustment } & \multicolumn{2}{|c|}{ Without adjustment } \\
\hline & & & & $\mathrm{OR}(95 \% \mathrm{Cl})$ & $\mathbf{P}$ & $\mathrm{OR}(95 \% \mathrm{Cl})$ & $\mathbf{P}$ \\
\hline \multirow[t]{5}{*}{ WLS } & rs2566755 & Homozygote & $\mathrm{CC} / \mathrm{TT}$ & $0.70(0.38-1.28)$ & 0.248 & $0.71(0.39-1.30)$ & 0.269 \\
\hline & & Heterozygote & $\mathrm{CT} / \mathrm{TT}$ & $0.84(0.64-1.10)$ & 0.205 & $0.84(0.64-1.10)$ & 0.194 \\
\hline & & Dominant & CC-CT/TT & $0.82(0.63-1.06)$ & 0.134 & $0.82(0.63-1.06)$ & 0.131 \\
\hline & & Recessive & CC/CT-TT & $0.74(0.41-1.35)$ & 0.328 & $0.76(0.42-1.37)$ & 0.356 \\
\hline & & Additive & & $0.84(0.68-1.04)$ & 0.109 & $0.84(0.68-1.04)$ & 0.111 \\
\hline \multirow[t]{5}{*}{ WLS } & rs12407028 & Homozygote & $\mathrm{CC} / \mathrm{TT}$ & $1.07(0.63-1.82)$ & 0.805 & $1.06(0.62-1.79)$ & 0.844 \\
\hline & & Heterozygote & $\mathrm{CT} / \mathrm{TT}$ & $1.03(0.79-1.33)$ & 0.847 & $1.00(0.77-1.30)$ & 0.980 \\
\hline & & Dominant & CC-CT/TT & $1.03(0.80-1.33)$ & 0.804 & $1.01(0.79-1.30)$ & 0.935 \\
\hline & & Recessive & CC/CT-TT & $1.06(0.63-1.79)$ & 0.830 & $1.05(0.63-1.78)$ & 0.845 \\
\hline & & Additive & & $1.03(0.84-1.26)$ & 0.775 & $1.02(0.83-1.24)$ & 0.887 \\
\hline \multirow[t]{5}{*}{ WLS } & rs2566752 & Homozygote & $\mathrm{TT} / \mathrm{CC}$ & $0.99(0.70-1.40)$ & 0.956 & $0.98(0.69-1.39)$ & 0.914 \\
\hline & & Heterozygote & $\mathrm{TC} / \mathrm{CC}$ & $1.02(0.76-1.35)$ & 0.920 & $0.99(0.74-1.31)$ & 0.917 \\
\hline & & Dominant & TT-TC/CC & $1.01(0.77-1.32)$ & 0.958 & $0.98(0.75-1.28)$ & 0.904 \\
\hline & & Recessive & TT/TC-CC & $0.98(0.73-1.33)$ & 0.902 & $0.99(0.73-1.34)$ & 0.948 \\
\hline & & Additive & & $1.00(0.84-1.18)$ & 0.971 & $0.99(0.83-1.18)$ & 0.908 \\
\hline \multirow[t]{5}{*}{ WLS } & rs7554551 & Homozygote & $\mathrm{CC} / \mathrm{TT}$ & $0.99(0.52-1.91)$ & 0.986 & $0.93(0.49-1.77)$ & 0.823 \\
\hline & & Heterozygote & $\mathrm{CT} / \mathrm{TT}$ & $0.95(0.73-1.23)$ & 0.678 & $0.97(0.75-1.26)$ & 0.833 \\
\hline & & Dominant & CC-CT/TT & $0.95(0.74-1.22)$ & 0.696 & $0.97(0.75-1.24)$ & 0.800 \\
\hline & & Recessive & CC/CT-TT & $1.01(0.53-1.93)$ & 0.968 & $0.94(0.49-1.78)$ & 0.846 \\
\hline & & Additive & & $0.96(0.78-1.20)$ & 0.747 & $0.97(0.78-1.20)$ & 0.777 \\
\hline \multirow[t]{5}{*}{ WNT16 } & rs3779381 & Homozygote & $\mathrm{GG} / \mathrm{AA}$ & $2.07(0.52-8.15)$ & 0.299 & $2.44(0.63-9.48)$ & 0.200 \\
\hline & & Heterozygote & GA/AA & $1.22(0.90-1.65)$ & 0.195 & $1.22(0.90-1.65)$ & 0.192 \\
\hline & & Dominant & GG-GA/AA & $1.25(0.93-1.68)$ & 0.145 & $1.26(0.94-1.69)$ & 0.129 \\
\hline & & Recessive & GG/GA-AA & $1.98(0.50-7.80)$ & 0.328 & $2.33(0.60-9.07)$ & 0.221 \\
\hline & & Additive & & $1.25(0.95-1.66)$ & 0.114 & $1.27(0.96-1.68)$ & 0.090 \\
\hline \multirow[t]{5}{*}{ WNT16 } & rs3801387 & Homozygote & GG/AA & $2.06(0.52-8.13)$ & 0.301 & $2.42(0.62-9.44)$ & 0.202 \\
\hline & & Heterozygote & GA/AA & $1.21(0.90-1.64)$ & 0.210 & $1.22(0.90-1.65)$ & 0.203 \\
\hline & & Dominant & GG-GA/AA & $1.24(0.92-1.67)$ & 0.157 & $1.25(0.93-1.69)$ & 0.138 \\
\hline & & Recessive & GG/GA-AA & $1.98(0.50-7.79)$ & 0.329 & $2.32(0.60-9.04)$ & 0.224 \\
\hline & & Additive & & $1.25(0.94-1.65)$ & 0.123 & $1.27(0.96-1.67)$ & 0.096 \\
\hline \multirow[t]{5}{*}{ WNT16 } & rs917727 & Homozygote & $\mathrm{TT} / \mathrm{CC}$ & $1.26(0.39-4.05)$ & 0.697 & $1.44(0.45-4.56)$ & 0.540 \\
\hline & & Heterozygote & $\mathrm{TC} / \mathrm{CC}$ & $1.15(0.84-1.57)$ & 0.376 & $1.15(0.85-1.57)$ & 0.364 \\
\hline & & Dominant & $\mathrm{TT}-\mathrm{TC} / \mathrm{CC}$ & $1.16(0.85-1.56)$ & 0.349 & $1.17(0.86-1.58)$ & 0.314 \\
\hline & & Recessive & TT/TC-CC & $1.23(0.38-3.93)$ & 0.732 & $1.39(0.44-4.42)$ & 0.572 \\
\hline & & Additive & & $1.14(0.87-1.51)$ & 0.344 & $1.16(0.88-1.53)$ & 0.287 \\
\hline
\end{tabular}

SNP, single nucleotide polymorphism; OR, odds ratio; $\mathrm{Cl}$, confidence interval.

Bold values mean statistical significance. 


\begin{tabular}{|c|c|c|c|c|c|c|c|}
\hline \multirow[t]{2}{*}{ Gene } & \multirow[t]{2}{*}{ SNP } & \multirow[t]{2}{*}{ Model } & \multirow[t]{2}{*}{ Genotype } & \multicolumn{2}{|l|}{ With adjustment } & \multicolumn{2}{|c|}{ Without adjustment } \\
\hline & & & & OR(95\% Cl) & $\mathbf{P}$ & OR(95\%Cl) & $\mathbf{P}$ \\
\hline \multirow[t]{5}{*}{ WNT16 } & rs7776725 & Homozygote & $\mathrm{CC} / \mathrm{TT}$ & $2.05(0.52-8.07)$ & 0.306 & $2.41(0.62-9.39)$ & 0.205 \\
\hline & & Heterozygote & $\mathrm{CT} / \mathrm{TT}$ & $1.17(0.86-1.58)$ & 0.320 & $1.17(0.86-1.58)$ & 0.315 \\
\hline & & Dominant & CC-CT/TT & $1.19(0.89-1.61)$ & 0.245 & $1.21(0.89-1.62)$ & 0.221 \\
\hline & & Recessive & CC/CT-TT & $1.98(0.50-7.80)$ & 0.328 & $2.33(0.60-9.07)$ & 0.221 \\
\hline & & Additive & & $1.21(0.91-1.60)$ & 0.192 & $1.23(0.93-1.62)$ & 0.155 \\
\hline \multirow[t]{5}{*}{ LRP5 } & rs2291467 & Homozygote & $\mathrm{TT} / \mathrm{CC}$ & $0.17(0.07-0.45)$ & $<0.001$ & $0.17(0.06-0.44)$ & $<0.001$ \\
\hline & & Heterozygote & $\mathrm{TC} / \mathrm{CC}$ & $0.97(0.75-1.27)$ & 0.838 & $0.97(0.74-1.26)$ & 0.794 \\
\hline & & Dominant & TT-TC/CC & $0.86(0.66-1.11)$ & 0.243 & $0.85(0.66-1.10)$ & 0.213 \\
\hline & & Recessive & TT/TC-CC & $0.17(0.07-0.45)$ & $<0.001$ & $0.17(0.06-0.44)$ & $<0.001$ \\
\hline & & Additive & & $0.77(0.61-0.96)$ & 0.021 & $0.76(0.61-0.95)$ & 0.017 \\
\hline \multirow[t]{5}{*}{ LRP5 } & rs11228240 & Homozygote & $\mathrm{TT} / \mathrm{CC}$ & $0.17(0.07-0.45)$ & $<0.001$ & $0.17(0.06-0.44)$ & $<0.001$ \\
\hline & & Heterozygote & $\mathrm{TC} / \mathrm{CC}$ & $0.98(0.75-1.27)$ & 0.875 & $0.97(0.75-1.26)$ & 0.818 \\
\hline & & Dominant & $\mathrm{TT}-\mathrm{TC} / \mathrm{CC}$ & $0.87(0.67-1.12)$ & 0.272 & $0.86(0.67-1.10)$ & 0.232 \\
\hline & & Recessive & TT/TC-CC & $0.17(0.07-0.46)$ & $<0.001$ & $0.17(0.06-0.44)$ & $<0.001$ \\
\hline & & Additive & & $0.77(0.62-0.97)$ & 0.025 & $0.77(0.61-0.96)$ & 0.019 \\
\hline \multirow[t]{5}{*}{ LRP5 } & rs12272917 & Homozygote & $\mathrm{CC} / \mathrm{TT}$ & $0.23(0.10-0.54)$ & 0.001 & $0.22(0.10-0.52)$ & $<0.001$ \\
\hline & & Heterozygote & $\mathrm{CT} / \mathrm{TT}$ & $0.92(0.71-1.20)$ & 0.560 & $0.92(0.71-1.20)$ & 0.552 \\
\hline & & Dominant & CC-CT/TT & $0.82(0.64-1.07)$ & 0.139 & $0.82(0.64-1.06)$ & 0.128 \\
\hline & & Recessive & CC/CT-TT & $0.24(0.10-0.55)$ & 0.001 & $0.23(0.10-0.53)$ & 0.001 \\
\hline & & Additive & & $0.76(0.60-0.94)$ & 0.013 & $0.75(0.60-0.94)$ & 0.011 \\
\hline \multicolumn{8}{|c|}{ SNP, single nucleotide polymorphism; OR, odds ratio; $\mathrm{Cl}$, confidence interval. } \\
\hline
\end{tabular}

We then performed stratification analysis of the genetic polymorphisms with osteoporosis risk (Table 3). We found that rs2291467, rs 11228240 and rs12272917 significantly decreased osteoporosis risk in the subgroup of age $>60$ years (rs2291467: homozygote, OR $=0.22,95 \% \mathrm{Cl}=0.07-$ $0.67, P=0.007$; recessive, $\mathrm{OR}=0.22,95 \% \mathrm{Cl}=0.07-0.67, P=0.008 ;$ rs 11228240 : homozygote, $\mathrm{OR}=0.22,95 \% \mathrm{Cl}=0.07-0.67, P=0.008$ and recessive, $\mathrm{OR}=0.22,95 \% \mathrm{Cl}=0.07-0.67, P=0.008 ;$ rs 12272917 : homozygote, $\mathrm{OR}=0.26,95 \% \mathrm{Cl}=0.09-0.72, P=0.010$ and recessive, $\mathrm{OR}=0.26$, $95 \% \mathrm{Cl}=0.10-0.73, P=0.010)$ and age $\leq 60$ years (rs2291467: homozygote, $\mathrm{OR}=0.00,95 \% \mathrm{Cl}=0.01-0.90, P=0.039$ and recessive, $\mathrm{OR}=0.12$, $95 \% \mathrm{Cl}=0.02-0.95, P=0.044 ;$ rs 11228240 : homozygote, $\mathrm{OR}=0.11,95 \% \mathrm{Cl}=0.01-0.89, P=0.039$ and recessive, $\mathrm{OR}=0.12,95 \% \mathrm{Cl}=0.02-0.95, P=$ 0.045 , additive, $\mathrm{OR}=0.65,95 \% \mathrm{Cl}=0.43-0.99, P=0.043 ;$ rs 12272917 : homozygote, $\mathrm{OR}=0.21,95 \% \mathrm{Cl}=0.05-0.97, P=0.046$ and recessive, $\mathrm{OR}=$ $0.66,95 \% \mathrm{Cl}=0.44-0.99, P=0.044)$. For individuals with higher $\mathrm{BMI}(\mathrm{BMI}>24)$, WNT16 polymorphisms (rs3779381, rs3801387, rs9117727 and rs7776725) were significantly associated with a higher risk of osteoporosis in multiple models $(P<0.05)$. In addition, $L R P 5$ polymorphisms (rs2291467, rs11228240 and rs12272917) had a strong relationship with an increased risk of osteoporosis among individuals with lower BMI $(\mathrm{BMI} \leq 24)$. 
Table 3

Stratification analysis of genetic polymorphism with osteoporosis risk

\begin{tabular}{|c|c|c|c|c|c|c|c|c|c|c|}
\hline \multirow[t]{2}{*}{ Gene } & \multirow[t]{2}{*}{ SNP } & \multirow[t]{2}{*}{ Model } & \multicolumn{2}{|l|}{ Age $>60$} & \multicolumn{2}{|l|}{ Age $\leq 60$} & \multicolumn{2}{|l|}{$\mathrm{BMI}>24$} & \multicolumn{2}{|l|}{$\mathrm{BMI} \leq 24$} \\
\hline & & & OR(95\%Cl) & $\mathbf{P}$ & OR(95\%Cl) & $\mathbf{P}$ & OR(95\%Cl) & $\mathbf{P}$ & OR(95\% Cl) & $\mathbf{P}$ \\
\hline \multirow[t]{6}{*}{ WLS } & rs2566755 & Allele & $\begin{array}{l}0.81(0.62- \\
1.06)\end{array}$ & 0.123 & $\begin{array}{l}0.90(0.61- \\
1.31)\end{array}$ & 0.572 & $\begin{array}{l}0.65(0.91- \\
0.61)\end{array}$ & 1.359 & $\begin{array}{l}0.93(0.67- \\
1.28)\end{array}$ & 0.642 \\
\hline & & Homozygote & $\begin{array}{l}0.94(0.46- \\
1.92)\end{array}$ & 0.860 & $\begin{array}{l}0.23(0.05- \\
1.10)\end{array}$ & 0.065 & $\begin{array}{l}0.90(0.30- \\
2.71)\end{array}$ & 0.849 & $\begin{array}{l}1.01(0.37- \\
2.78)\end{array}$ & 0.978 \\
\hline & & Heterozygote & $\begin{array}{l}0.69(0.50- \\
0.97)\end{array}$ & 0.031 & $\begin{array}{l}1.04(0.64- \\
1.69)\end{array}$ & 0.873 & $\begin{array}{l}0.82(0.5- \\
1.36)\end{array}$ & 0.451 & $\begin{array}{l}0.89(0.60- \\
1.32)\end{array}$ & 0.564 \\
\hline & & Dominant & $\begin{array}{l}1.03(0.76- \\
1.41)\end{array}$ & 0.840 & $\begin{array}{l}0.91(0.57- \\
1.46)\end{array}$ & 0.705 & $\begin{array}{l}0.83(0.52- \\
1.35)\end{array}$ & 0.457 & $\begin{array}{l}0.90(0.61- \\
1.32)\end{array}$ & 0.596 \\
\hline & & Recessive & $\begin{array}{l}1.06(0.52- \\
2.14)\end{array}$ & 0.881 & $\begin{array}{l}0.23(0.05- \\
1.07)\end{array}$ & 0.061 & $\begin{array}{l}0.96(0.32- \\
2.85)\end{array}$ & 0.940 & $\begin{array}{l}1.05(0.39- \\
2.86)\end{array}$ & 0.921 \\
\hline & & Additive & $\begin{array}{l}0.81(0.62- \\
1.05)\end{array}$ & 0.109 & $\begin{array}{l}0.81(0.54- \\
1.22)\end{array}$ & 0.306 & $\begin{array}{l}0.88(0.59- \\
1.31)\end{array}$ & 0.519 & $\begin{array}{l}0.93(0.67- \\
1.29)\end{array}$ & 0.673 \\
\hline \multirow[t]{6}{*}{ WLS } & rs12407028 & Allele & $\begin{array}{l}0.97(0.76- \\
1.26)\end{array}$ & 0.838 & $\begin{array}{l}1.14(0.80- \\
1.62)\end{array}$ & 0.462 & $\begin{array}{l}0.67(1.09- \\
0.74)\end{array}$ & 1.593 & $\begin{array}{l}1.08(0.80- \\
1.47)\end{array}$ & 0.606 \\
\hline & & Homozygote & $\begin{array}{l}0.83(0.42- \\
1.65)\end{array}$ & 0.593 & $\begin{array}{l}1.47(0.60- \\
3.64)\end{array}$ & 0.401 & $\begin{array}{l}1.19(0.38- \\
3.70)\end{array}$ & 0.769 & $\begin{array}{l}1.39(0.62- \\
3.11)\end{array}$ & 0.426 \\
\hline & & Heterozygote & $\begin{array}{l}1.07(0.77- \\
1.48)\end{array}$ & 0.690 & $\begin{array}{l}1.14(0.70- \\
1.84)\end{array}$ & 0.601 & $\begin{array}{l}1.26(0.77- \\
2.05)\end{array}$ & 0.362 & $\begin{array}{l}1.00(0.68- \\
1.47)\end{array}$ & 0.998 \\
\hline & & Dominant & $\begin{array}{l}1.03(0.76- \\
1.41)\end{array}$ & 0.840 & $\begin{array}{l}1.19(0.75- \\
1.87)\end{array}$ & 0.466 & $\begin{array}{l}1.25(0.78- \\
2.00)\end{array}$ & 0.359 & $\begin{array}{l}1.05(0.73- \\
1.52)\end{array}$ & 0.800 \\
\hline & & Recessive & $\begin{array}{l}0.81(0.41- \\
1.60)\end{array}$ & 0.542 & $\begin{array}{l}1.40(0.58- \\
3.39)\end{array}$ & 0.455 & $\begin{array}{l}1.08(0.35- \\
3.30)\end{array}$ & 0.892 & $\begin{array}{l}1.39(0.63- \\
3.07)\end{array}$ & 0.419 \\
\hline & & Additive & $\begin{array}{l}0.99(0.77- \\
1.28)\end{array}$ & 0.950 & $\begin{array}{l}1.18(0.82- \\
1.69)\end{array}$ & 0.377 & $\begin{array}{l}1.19(0.79- \\
1.77)\end{array}$ & 0.408 & $\begin{array}{l}1.08(0.80- \\
1.46)\end{array}$ & 0.601 \\
\hline \multirow[t]{6}{*}{ WLS } & rs2566752 & Allele & $\begin{array}{l}0.99(0.80- \\
1.22)\end{array}$ & 0.906 & $\begin{array}{l}1.01(0.74- \\
1.37)\end{array}$ & 0.961 & $\begin{array}{l}0.46(1.13- \\
0.82)\end{array}$ & 1.557 & $\begin{array}{l}0.97(0.75- \\
1.25)\end{array}$ & 0.794 \\
\hline & & Homozygote & $\begin{array}{l}1.02(0.67- \\
1.56)\end{array}$ & 0.936 & $\begin{array}{l}1.13(0.58- \\
2.19)\end{array}$ & 0.715 & $\begin{array}{l}1.14(0.59- \\
2.18)\end{array}$ & 0.696 & $\begin{array}{l}0.91(0.54- \\
1.54)\end{array}$ & 0.735 \\
\hline & & Heterozygote & $\begin{array}{l}0.94(0.67- \\
1.34)\end{array}$ & 0.747 & $\begin{array}{l}1.47(0.86- \\
2.52)\end{array}$ & 0.162 & $\begin{array}{l}0.81(0.46- \\
1.43)\end{array}$ & 0.472 & $\begin{array}{l}1.03(0.68- \\
1.56)\end{array}$ & 0.892 \\
\hline & & Dominant & $\begin{array}{l}0.97(0.70- \\
1.34)\end{array}$ & 0.838 & $\begin{array}{l}1.36(0.82- \\
2.27)\end{array}$ & 0.237 & $\begin{array}{l}0.91(0.54- \\
1.56)\end{array}$ & 0.738 & $\begin{array}{l}0.99(0.67- \\
1.47)\end{array}$ & 0.978 \\
\hline & & Recessive & $\begin{array}{l}1.05(0.73- \\
1.53)\end{array}$ & 0.782 & $\begin{array}{l}0.88(0.50- \\
1.54)\end{array}$ & 0.654 & $\begin{array}{l}1.31(0.77- \\
2.22)\end{array}$ & 0.323 & $\begin{array}{l}0.90(0.57- \\
1.42)\end{array}$ & 0.645 \\
\hline & & Additive & $\begin{array}{l}1.00(0.81- \\
1.24)\end{array}$ & 0.979 & $\begin{array}{l}1.09(0.79- \\
1.50)\end{array}$ & 0.616 & $\begin{array}{l}1.07(0.78- \\
1.47)\end{array}$ & 0.686 & $\begin{array}{l}0.96(0.75- \\
1.25)\end{array}$ & 0.782 \\
\hline \multirow[t]{5}{*}{ WLS } & rs7554551 & Allele & $\begin{array}{l}0.97(0.75- \\
1.26)\end{array}$ & 0.842 & $\begin{array}{l}0.96(0.66- \\
1.39)\end{array}$ & 0.833 & $\begin{array}{l}0.26(1.25- \\
0.85)\end{array}$ & 1.849 & $\begin{array}{l}0.97(0.70- \\
1.33)\end{array}$ & 0.850 \\
\hline & & Homozygote & $\begin{array}{l}1.01(0.42- \\
2.45)\end{array}$ & 0.976 & $\begin{array}{l}0.77(0.27- \\
2.20)\end{array}$ & 0.627 & $\begin{array}{l}1.29(0.27- \\
6.19)\end{array}$ & 0.752 & $\begin{array}{l}1.02(0.42- \\
2.48)\end{array}$ & 0.967 \\
\hline & & Heterozygote & $\begin{array}{l}0.93(0.68- \\
1.28)\end{array}$ & 0.649 & $\begin{array}{l}0.86(0.52- \\
1.40)\end{array}$ & 0.535 & $\begin{array}{l}1.25(0.77- \\
2.03)\end{array}$ & 0.362 & $\begin{array}{l}0.94(0.64- \\
1.40)\end{array}$ & 0.777 \\
\hline & & Dominant & $\begin{array}{l}0.94(0.69- \\
1.28)\end{array}$ & 0.672 & $\begin{array}{l}0.84(0.53- \\
1.35)\end{array}$ & 0.477 & $\begin{array}{l}1.25(0.78- \\
2.01)\end{array}$ & 0.349 & $\begin{array}{l}0.95(0.65- \\
1.39)\end{array}$ & 0.806 \\
\hline & & Recessive & $\begin{array}{l}1.04(0.43- \\
2.50)\end{array}$ & 0.929 & $\begin{array}{l}0.81(0.29- \\
2.29)\end{array}$ & 0.694 & $\begin{array}{l}1.19(0.25- \\
5.69)\end{array}$ & 0.828 & $\begin{array}{l}1.04(0.43- \\
2.50)\end{array}$ & 0.935 \\
\hline
\end{tabular}

SNP, single nucleotide polymorphism; BMI, body mass index; OR, odds ratio; $\mathrm{Cl}$, confidence interval.

Bold values mean statistical significance. 


\begin{tabular}{|c|c|c|c|c|c|c|c|c|c|c|}
\hline \multirow[t]{2}{*}{ Gene } & \multirow[t]{2}{*}{ SNP } & \multirow[t]{2}{*}{ Model } & \multicolumn{2}{|l|}{ Age $>60$} & \multicolumn{2}{|l|}{ Age $\leq 60$} & \multicolumn{2}{|l|}{$\mathrm{BMI}>24$} & \multicolumn{2}{|l|}{$\mathrm{BMI} \leq 24$} \\
\hline & & & $\mathrm{OR}(95 \% \mathrm{Cl})$ & $\mathbf{P}$ & OR(95\%Cl) & $\mathbf{P}$ & $\mathrm{OR}(95 \% \mathrm{Cl})$ & $\mathbf{P}$ & OR(95\%Cl) & $P$ \\
\hline & & Additive & $\begin{array}{l}0.95(0.73- \\
1.25)\end{array}$ & 0.730 & $\begin{array}{l}0.87(0.59- \\
1.28)\end{array}$ & 0.465 & $\begin{array}{l}1.22(0.79- \\
1.88)\end{array}$ & 0.362 & $\begin{array}{l}0.97(0.71- \\
1.33)\end{array}$ & 0.860 \\
\hline \multirow[t]{6}{*}{ WNT16 } & rs3779381 & Allele & $\begin{array}{l}1.34(0.96- \\
1.87)\end{array}$ & 0.083 & $\begin{array}{l}1.08(0.66- \\
1.76)\end{array}$ & 0.760 & $\begin{array}{l}0.02(1.85- \\
1.09)\end{array}$ & 3.128 & $\begin{array}{l}1.19(0.78- \\
1.80)\end{array}$ & 0.417 \\
\hline & & Homozygote & $\begin{array}{l}1.92(0.48- \\
7.63)\end{array}$ & 0.356 & - & - & $\begin{array}{l}0.65(0.04- \\
10.92)\end{array}$ & 0.764 & - & - \\
\hline & & Heterozygote & $\begin{array}{l}1.32(0.90- \\
1.92)\end{array}$ & 0.154 & - & - & $\begin{array}{l}2.18(1.21- \\
3.92)\end{array}$ & 0.009 & $\begin{array}{l}1.01(0.64- \\
1.58)\end{array}$ & 0.977 \\
\hline & & Dominant & $\begin{array}{l}1.35(0.93- \\
1.94)\end{array}$ & 0.114 & $\begin{array}{l}1.31(0.76- \\
2.25)\end{array}$ & 0.338 & $\begin{array}{l}2.10(1.18- \\
3.74)\end{array}$ & 0.012 & $\begin{array}{l}1.09(0.70- \\
1.71)\end{array}$ & 0.698 \\
\hline & & Recessive & $\begin{array}{l}1.81(0.46- \\
7.20)\end{array}$ & 0.399 & - & - & $\begin{array}{l}0.55(0.03- \\
9.24)\end{array}$ & 0.679 & - & - \\
\hline & & Additive & $\begin{array}{l}1.33(0.95- \\
1.86)\end{array}$ & 0.098 & $\begin{array}{l}1.31(0.76- \\
2.25)\end{array}$ & 0.338 & $\begin{array}{l}1.95(1.12- \\
3.41)\end{array}$ & 0.019 & $\begin{array}{l}1.18(0.78- \\
1.80)\end{array}$ & 0.441 \\
\hline \multirow[t]{6}{*}{ WNT16 } & rs3801387 & Allele & $\begin{array}{l}1.31(0.94- \\
1.83)\end{array}$ & 0.110 & $\begin{array}{l}1.11(0.68- \\
1.82)\end{array}$ & 0.672 & $\begin{array}{l}0.02(1.84- \\
1.09)\end{array}$ & 3.123 & $\begin{array}{l}1.22(0.80- \\
1.86)\end{array}$ & 0.357 \\
\hline & & Homozygote & $\begin{array}{l}1.90(0.48- \\
7.56)\end{array}$ & 0.363 & - & - & $\begin{array}{l}0.65(0.04- \\
10.91)\end{array}$ & 0.763 & - & - \\
\hline & & Heterozygote & $\begin{array}{l}1.28(0.88- \\
1.86)\end{array}$ & 0.202 & - & - & $\begin{array}{l}2.18(1.21- \\
3.92)\end{array}$ & 0.009 & $\begin{array}{l}1.04(0.66- \\
1.63)\end{array}$ & 0.883 \\
\hline & & Dominant & $\begin{array}{l}1.31(0.91- \\
1.89)\end{array}$ & 0.151 & $\begin{array}{l}1.38(0.80- \\
2.38)\end{array}$ & 0.253 & $\begin{array}{l}2.10(1.18- \\
3.74)\end{array}$ & 0.012 & $\begin{array}{l}1.12(0.71- \\
1.77)\end{array}$ & 0.614 \\
\hline & & Recessive & $\begin{array}{l}1.81(0.45- \\
7.17)\end{array}$ & 0.401 & - & - & $\begin{array}{l}0.55(0.03- \\
9.22)\end{array}$ & 0.677 & - & - \\
\hline & & Additive & $\begin{array}{l}1.30(0.93- \\
1.82)\end{array}$ & 0.128 & $\begin{array}{l}1.38(0.80- \\
2.38)\end{array}$ & 0.253 & $\begin{array}{l}1.95(1.12- \\
3.41)\end{array}$ & 0.019 & $\begin{array}{l}1.21(0.79- \\
1.85)\end{array}$ & 0.380 \\
\hline \multirow[t]{6}{*}{ WNT16 } & rs917727 & Allele & $\begin{array}{l}1.20(0.85- \\
1.68)\end{array}$ & 0.299 & $\begin{array}{l}1.08(0.66- \\
1.76)\end{array}$ & 0.760 & $\begin{array}{l}0.09(1.57- \\
0.93)\end{array}$ & 2.627 & $\begin{array}{l}1.18(0.77- \\
1.80)\end{array}$ & 0.460 \\
\hline & & Homozygote & $\begin{array}{l}1.43(0.41- \\
5.05)\end{array}$ & 0.575 & - & - & $\begin{array}{l}0.26(0.03- \\
2.61)\end{array}$ & 0.253 & - & - \\
\hline & & Heterozygote & $\begin{array}{l}1.16(0.79- \\
1.70)\end{array}$ & 0.447 & $\begin{array}{l}1.39(0.80- \\
2.41)\end{array}$ & 0.238 & $\begin{array}{l}2.17(1.18- \\
3.98)\end{array}$ & 0.012 & $\begin{array}{l}0.98(0.62- \\
1.56)\end{array}$ & 0.937 \\
\hline & & Dominant & $\begin{array}{l}1.18(0.81- \\
1.71)\end{array}$ & 0.388 & $\begin{array}{l}1.37(0.79- \\
2.36)\end{array}$ & 0.264 & $\begin{array}{l}1.89(1.06- \\
3.38)\end{array}$ & 0.031 & $\begin{array}{l}1.07(0.68- \\
1.70)\end{array}$ & 0.763 \\
\hline & & Recessive & $\begin{array}{l}1.39(0.40- \\
4.89)\end{array}$ & 0.606 & - & - & $\begin{array}{l}0.22(0.02- \\
2.23)\end{array}$ & 0.201 & - & - \\
\hline & & Additive & $\begin{array}{l}1.17(0.84- \\
1.64)\end{array}$ & 0.360 & $\begin{array}{l}1.32(0.77- \\
2.26)\end{array}$ & 0.309 & $\begin{array}{l}1.55(0.92- \\
2.64)\end{array}$ & 0.102 & $\begin{array}{l}1.17(0.76- \\
1.79)\end{array}$ & 0.485 \\
\hline \multirow[t]{5}{*}{ WNT16 } & rs7776725 & Allele & $\begin{array}{l}1.25(0.89- \\
1.75)\end{array}$ & 0.198 & $\begin{array}{l}1.15(0.71- \\
1.87)\end{array}$ & 0.561 & $\begin{array}{l}0.03(1.81- \\
1.07)\end{array}$ & 3.062 & $\begin{array}{l}1.17(0.77- \\
1.79)\end{array}$ & 0.459 \\
\hline & & Homozygote & $\begin{array}{l}1.87(0.47- \\
7.46)\end{array}$ & 0.373 & - & - & $\begin{array}{l}0.64(0.04- \\
10.84)\end{array}$ & 0.760 & - & - \\
\hline & & Heterozygote & $\begin{array}{l}1.18(0.81- \\
1.73)\end{array}$ & 0.393 & - & - & $\begin{array}{l}2.10(1.17- \\
3.77)\end{array}$ & 0.013 & $\begin{array}{l}0.99(0.62- \\
1.56)\end{array}$ & 0.949 \\
\hline & & Dominant & $\begin{array}{l}1.22(0.84- \\
1.76)\end{array}$ & 0.304 & $\begin{array}{l}1.45(0.84- \\
2.49)\end{array}$ & 0.178 & $\begin{array}{l}2.02(1.14- \\
3.60)\end{array}$ & 0.017 & $\begin{array}{l}1.07(0.68- \\
1.69)\end{array}$ & 0.757 \\
\hline & & Recessive & $\begin{array}{l}1.81(0.46- \\
7.20)\end{array}$ & 0.399 & - & - & $\begin{array}{l}0.55(0.03- \\
9.24)\end{array}$ & 0.679 & - & - \\
\hline
\end{tabular}

SNP, single nucleotide polymorphism; BMI, body mass index; OR, odds ratio; $\mathrm{Cl}$, confidence interval.

Bold values mean statistical significance. 


\begin{tabular}{|c|c|c|c|c|c|c|c|c|c|c|}
\hline \multirow[t]{2}{*}{ Gene } & \multirow[t]{2}{*}{ SNP } & \multirow[t]{2}{*}{ Model } & \multicolumn{2}{|l|}{ Age $>60$} & \multicolumn{2}{|l|}{ Age $\leq 60$} & \multicolumn{2}{|l|}{$\mathrm{BMI}>24$} & \multicolumn{2}{|l|}{$\mathrm{BMI} \leq 24$} \\
\hline & & & $\mathrm{OR}(95 \% \mathrm{Cl})$ & $\mathbf{P}$ & $\mathrm{OR}(95 \% \mathrm{Cl})$ & $\mathbf{P}$ & OR(95\%Cl) & $\mathbf{P}$ & $\mathrm{OR}(95 \% \mathrm{Cl})$ & $\mathbf{P}$ \\
\hline & & Additive & $\begin{array}{l}1.22(0.87- \\
1.71)\end{array}$ & 0.248 & $\begin{array}{l}1.45(0.84- \\
2.49)\end{array}$ & 0.178 & $\begin{array}{l}1.88(1.08- \\
3.28)\end{array}$ & 0.027 & $\begin{array}{l}1.16(0.76- \\
1.78)\end{array}$ & 0.483 \\
\hline \multirow[t]{6}{*}{ LRP5 } & rs2291467 & Allele & $\begin{array}{l}0.79(0.60- \\
1.04)\end{array}$ & 0.089 & $\begin{array}{l}0.75(0.52- \\
1.10)\end{array}$ & 0.136 & $\begin{array}{l}0.99(1.00- \\
0.67)\end{array}$ & 1.494 & $\begin{array}{l}0.67(0.49- \\
0.92)\end{array}$ & 0.013 \\
\hline & & Homozygote & $\begin{array}{l}0.22(0.07- \\
0.67)\end{array}$ & 0.007 & $\begin{array}{l}0.11(0.01- \\
0.90)\end{array}$ & 0.039 & $\begin{array}{l}0.27(0.05- \\
1.37)\end{array}$ & 0.113 & $\begin{array}{l}0.14(0.04- \\
0.53)\end{array}$ & 0.004 \\
\hline & & Heterozygote & $\begin{array}{l}0.96(0.69- \\
1.33)\end{array}$ & 0.805 & $\begin{array}{l}0.83(0.51- \\
1.35)\end{array}$ & 0.458 & $\begin{array}{l}1.33(0.81- \\
2.17)\end{array}$ & 0.264 & $\begin{array}{l}0.80(0.55- \\
1.19)\end{array}$ & 0.272 \\
\hline & & Dominant & $\begin{array}{l}0.86(0.63- \\
1.18)\end{array}$ & 0.352 & $\begin{array}{l}0.72(0.45- \\
1.16)\end{array}$ & 0.179 & $\begin{array}{l}1.19(0.73- \\
1.91)\end{array}$ & 0.486 & $\begin{array}{l}0.71(0.49- \\
1.04)\end{array}$ & 0.077 \\
\hline & & Recessive & $\begin{array}{l}0.22(0.07- \\
0.67)\end{array}$ & 0.008 & $\begin{array}{l}0.12(0.02- \\
0.95)\end{array}$ & 0.044 & $\begin{array}{l}0.24(0.05- \\
1.23)\end{array}$ & 0.087 & $\begin{array}{l}0.15(0.04- \\
0.57)\end{array}$ & 0.005 \\
\hline & & Additive & $\begin{array}{l}0.78(0.59- \\
1.04)\end{array}$ & 0.086 & $\begin{array}{l}0.66(0.44- \\
1.00)\end{array}$ & 0.051 & $\begin{array}{l}1.02(0.66- \\
1.55)\end{array}$ & 0.943 & $\begin{array}{l}0.65(0.47- \\
0.91)\end{array}$ & 0.012 \\
\hline \multirow[t]{6}{*}{ LRP5 } & rs11228240 & Allele & $\begin{array}{l}0.80(0.61- \\
1.05)\end{array}$ & 0.104 & $\begin{array}{l}0.75(0.51- \\
1.09)\end{array}$ & 0.125 & $\begin{array}{l}0.83(0.96- \\
0.65)\end{array}$ & 1.422 & $\begin{array}{l}0.69(0.5- \\
0.94)\end{array}$ & 0.020 \\
\hline & & Homozygote & $\begin{array}{l}0.22(0.07- \\
0.67)\end{array}$ & 0.008 & $\begin{array}{l}0.11(0.01- \\
0.89)\end{array}$ & 0.039 & $\begin{array}{l}0.26(0.05- \\
1.35)\end{array}$ & 0.109 & $\begin{array}{l}0.14(0.04- \\
0.54)\end{array}$ & 0.004 \\
\hline & & Heterozygote & $\begin{array}{l}0.98(0.71- \\
1.35)\end{array}$ & 0.894 & $\begin{array}{l}0.81(0.50- \\
1.32)\end{array}$ & 0.404 & $\begin{array}{l}1.23(0.76- \\
2.01)\end{array}$ & 0.400 & $\begin{array}{l}0.83(0.56- \\
1.22)\end{array}$ & 0.344 \\
\hline & & Dominant & $\begin{array}{l}0.88(0.64- \\
1.21)\end{array}$ & 0.426 & $\begin{array}{l}0.71(0.44- \\
1.14)\end{array}$ & 0.153 & $\begin{array}{l}1.11(0.69- \\
1.79)\end{array}$ & 0.659 & $\begin{array}{l}0.74(0.51- \\
1.07)\end{array}$ & 0.108 \\
\hline & & Recessive & $\begin{array}{l}0.22(0.07- \\
0.67)\end{array}$ & 0.008 & $\begin{array}{l}0.12(0.02- \\
0.95)\end{array}$ & 0.045 & $\begin{array}{l}0.24(0.05- \\
1.24)\end{array}$ & 0.088 & $\begin{array}{l}0.15(0.04- \\
0.57)\end{array}$ & 0.005 \\
\hline & & Additive & $\begin{array}{l}0.80(0.60- \\
1.05)\end{array}$ & 0.111 & $\begin{array}{l}0.65(0.43- \\
0.99)\end{array}$ & 0.043 & $\begin{array}{l}0.97(0.63- \\
1.48)\end{array}$ & 0.881 & $\begin{array}{l}0.67(0.48- \\
0.93)\end{array}$ & 0.018 \\
\hline \multirow[t]{6}{*}{ LRP5 } & rs12272917 & Allele & $\begin{array}{l}0.79(0.61- \\
1.04)\end{array}$ & 0.091 & $\begin{array}{l}0.70(0.48- \\
1.02)\end{array}$ & 0.062 & $\begin{array}{l}0.68(0.92- \\
0.62)\end{array}$ & 1.365 & $\begin{array}{l}0.69(0.50- \\
0.95)\end{array}$ & 0.021 \\
\hline & & Homozygote & $\begin{array}{l}0.26(0.09- \\
0.72)\end{array}$ & 0.010 & $\begin{array}{l}0.21(0.05- \\
0.97)\end{array}$ & 0.046 & $\begin{array}{l}0.30(0.08- \\
1.17)\end{array}$ & 0.084 & $\begin{array}{l}0.21(0.06- \\
0.71)\end{array}$ & 0.012 \\
\hline & & Heterozygote & $\begin{array}{l}0.96(0.70- \\
1.33)\end{array}$ & 0.824 & $\begin{array}{l}0.78(0.48- \\
1.27)\end{array}$ & 0.313 & $\begin{array}{l}1.20(0.73- \\
1.97)\end{array}$ & 0.471 & $\begin{array}{l}0.78(0.53- \\
1.15)\end{array}$ & 0.217 \\
\hline & & Dominant & $\begin{array}{l}0.87(0.63- \\
1.19)\end{array}$ & 0.374 & $\begin{array}{l}0.69(0.43- \\
1.11)\end{array}$ & 0.124 & $\begin{array}{l}1.05(0.65- \\
1.69)\end{array}$ & 0.839 & $\begin{array}{l}0.71(0.49- \\
1.04)\end{array}$ & 0.077 \\
\hline & & Recessive & $\begin{array}{l}0.26(0.10- \\
0.73)\end{array}$ & 0.010 & $\begin{array}{l}0.23(0.05- \\
1.05)\end{array}$ & 0.057 & $\begin{array}{l}0.28(0.07- \\
1.09)\end{array}$ & 0.067 & $\begin{array}{l}0.23(0.07- \\
0.76)\end{array}$ & 0.016 \\
\hline & & Additive & $\begin{array}{l}0.79(0.60- \\
1.04)\end{array}$ & 0.098 & $\begin{array}{l}0.66(0.44- \\
0.99)\end{array}$ & 0.044 & $\begin{array}{l}0.91(0.60- \\
1.36)\end{array}$ & 0.634 & $\begin{array}{l}0.67(0.48- \\
0.94)\end{array}$ & 0.019 \\
\hline \multicolumn{11}{|c|}{ SNP, single nucleotide polymorphism; BMI, body mass index; OR, odds ratio; $\mathrm{Cl}$, confidence interval. } \\
\hline & & & & & & & & & & \\
\hline
\end{tabular}

We further conducted haplotype analysis of the genetic polymorphisms with osteoporosis risk (Table 4, Fig. 1-3). We observed three blocks: block 1 (rs2566755 and rs12407028), block 2 (rs3779381, rs3801387, rs917727 and rs7776725) and block 3 (rs2291467 and rs11228240). As shown in Table 4, the haplotype $\mathrm{T}_{\mathrm{rs} 2291467} \mathrm{~T}_{\mathrm{rs} 11228240}$ (with adjustment: $\mathrm{OR}=0.78,95 \% \mathrm{Cl}=0.62-0.97, P=0.028$; without adjustment: $\mathrm{OR}=0.77,95 \% \mathrm{Cl}=$ $0.62-0.96, P=0.022$ ) and $\mathrm{C}_{\mathrm{rs} 2291467} \mathrm{C}_{\mathrm{rs} 11228240}$ (with adjustment: $\mathrm{OR}=0.77,95 \% \mathrm{Cl}=0.62-0.96, P=0.023$; without adjustment: $\mathrm{OR}=0.76,95 \% \mathrm{Cl}=$ $0.61-0.95, P=0.017)$ could protect individuals with osteoporosis. 
Table 4

Haplotype analysis of genetic polymorphism with osteoporosis risk

\begin{tabular}{|c|c|c|c|c|c|c|c|c|}
\hline \multirow[t]{2}{*}{ Gene } & \multirow[t]{2}{*}{ SNP } & \multirow[t]{2}{*}{ Haplotype } & \multirow{2}{*}{$\begin{array}{l}\text { Frequency } \\
\text { in case }\end{array}$} & \multirow{2}{*}{$\begin{array}{l}\text { Frequency } \\
\text { in control }\end{array}$} & \multicolumn{2}{|c|}{ With adjustment } & \multicolumn{2}{|c|}{ Without adjustment } \\
\hline & & & & & $\mathrm{OR}(95 \% \mathrm{Cl})$ & $\mathbf{P}$ & $\mathrm{OR}(95 \% \mathrm{Cl})$ & $\mathbf{P}$ \\
\hline WLS & rs2566755|rs12407028 & $\mathrm{TC}$ & 0.241 & 0.238 & $\begin{array}{l}1.03(0.84- \\
1.27)\end{array}$ & 0.767 & $\begin{array}{l}1.02(0.83- \\
1.25)\end{array}$ & 0.869 \\
\hline WLS & rs2566755|rs12407028 & CT & 0.189 & 0.220 & $\begin{array}{l}0.83(0.67- \\
1.03)\end{array}$ & 0.087 & $\begin{array}{l}0.83(0.67- \\
1.03)\end{array}$ & 0.088 \\
\hline WLS & rs2566755|rs12407028 & TT & 0.430 & 0.458 & $\begin{array}{l}0.90(0.76- \\
1.08)\end{array}$ & 0.254 & $\begin{array}{l}0.90(0.75- \\
1.07)\end{array}$ & 0.213 \\
\hline WNT16 & rs3779381|rs3801387|rs917727|rs7776725 & GGTC & 0.117 & 0.101 & $\begin{array}{l}1.16(0.88- \\
1.55)\end{array}$ & 0.296 & $\begin{array}{l}1.18(0.89- \\
1.57)\end{array}$ & 0.248 \\
\hline WNT16 & rs3779381|rs3801387|rs917727|rs7776725 & AACT & 0.130 & 0.106 & $\begin{array}{l}1.25(0.95- \\
1.65)\end{array}$ & 0.106 & $\begin{array}{l}1.27(0.97- \\
1.67)\end{array}$ & 0.084 \\
\hline LRP5 & rs2291467|rs11228240 & TT & 0.177 & 0.216 & $\begin{array}{l}0.78(0.62- \\
0.97)\end{array}$ & 0.028 & $\begin{array}{l}0.77(0.62- \\
0.96)\end{array}$ & 0.022 \\
\hline LRP5 & rs2291467|rs11228240 & $\mathrm{CC}$ & 0.182 & 0.223 & $\begin{array}{l}0.77(0.62- \\
0.96)\end{array}$ & 0.023 & $\begin{array}{l}0.76(0.61- \\
0.95)\end{array}$ & 0.017 \\
\hline \multicolumn{9}{|c|}{ SNP, single nucleotide polymorphism; OR, odds ratio; $\mathrm{Cl}$, confidence interval. } \\
\hline$d_{1}$ & nean statistical signi & & & & & & & \\
\hline
\end{tabular}

Association of genetic polymorphisms and clinical indicators

In Table 5 and Supplemental Table 3, we evaluated the association of the genetic polymorphisms and clinical indicators (UA, urea, fasting glucose, WBC, RBC, PLT, PDW, MPV, PCT, L1, L2, L3 and L4) among the osteoporosis patients. The results revealed that the different genotypes of WLS, rs2566755, WNT16 rs917727, WNT16 rs7776725, LRP rs2291467, LRP rs11228240 and $L R P$ rs12272917, had significant differences in the BMD of L1-L4 $(P<0.05)$. The genotypes of WLS rs2566752 were not only related to L1 $(P=0.006)$ but were also associated with the levels of PDW $(P=$ $0.010)$ and MPV $(P=0.007)$. WLS rs7554551 also significantly influenced PLT $(P=0.010)$, PDW $(P=0.017), \mathrm{MPV}(P=0.011)$ and PCT $(P=0.045)$ levels. There were no significant relationships between WLS rs12407028, WNT16 rs3779381 and WNT16 rs3801387 and clinical indicators $(P>$ $0.05)$. 
Table 5

The relationships of clinical indicators and genetic polymorphism

\begin{tabular}{|c|c|c|c|c|c|c|c|c|}
\hline \multirow{2}{*}{$\begin{array}{l}\text { Clinical } \\
\text { indicators }\end{array}$} & \multicolumn{3}{|c|}{ WLS-rs2566755 } & \multicolumn{3}{|c|}{ WLS-rs2566752 } & \multirow[b]{2}{*}{$\mathrm{CC}$} & \multirow[b]{2}{*}{$\mathbf{P}$} \\
\hline & TT & CT & $\mathrm{CC}$ & $\mathbf{P}$ & TT & CT & & \\
\hline UA & $\begin{array}{l}264.41 \pm \\
91.54\end{array}$ & $\begin{array}{l}255.34 \pm \\
72.80\end{array}$ & $\begin{array}{l}254.15 \pm \\
67.38\end{array}$ & 0.517 & $\begin{array}{l}254.83 \pm \\
82.09\end{array}$ & $\begin{array}{l}265.55 \pm \\
87.71\end{array}$ & $257.05 \pm 83.40$ & 0.448 \\
\hline Urea & $5.88 \pm 2.12$ & $5.92 \pm 1.85$ & $6.00 \pm 1.98$ & 0.953 & $6.02 \pm 2.36$ & $5.95 \pm 2.04$ & $5.70 \pm 1.77$ & 0.369 \\
\hline Fasting glucose & $4.76 \pm 1.16$ & $4.77 \pm 1.29$ & $4.68 \pm 0.76$ & 0.949 & $4.84 \pm 1.60$ & $4.73 \pm 0.91$ & $4.78 \pm 1.29$ & 0.701 \\
\hline WBC & $7.24 \pm 2.53$ & $7.42 \pm 2.83$ & $6.96 \pm 2.25$ & 0.659 & $7.29 \pm 2.65$ & $7.29 \pm 2.54$ & $7.35 \pm 2.75$ & 0.969 \\
\hline $\mathrm{RBC}$ & $4.35 \pm 0.54$ & $4.30 \pm 0.52$ & $4.27 \pm 0.49$ & 0.560 & $4.33 \pm 0.60$ & $4.33 \pm 0.51$ & $4.31 \pm 0.53$ & 0.901 \\
\hline PLT & $\begin{array}{l}254.46 \pm \\
99.20\end{array}$ & $\begin{array}{l}267.92 \pm \\
112.24\end{array}$ & $\begin{array}{l}243.70 \pm \\
91.91\end{array}$ & 0.335 & $\begin{array}{l}253.10 \pm \\
99.27\end{array}$ & $\begin{array}{l}256.93 \pm \\
102.58\end{array}$ & $\begin{array}{l}266.19 \pm \\
107.92\end{array}$ & 0.545 \\
\hline PDW & $12.90 \pm 3.38$ & $12.15 \pm 3.85$ & $12.73 \pm 3.37$ & 0.300 & $13.11 \pm 3.36$ & $12.94 \pm 3.44$ & $12.02 \pm 3.19$ & 0.010 \\
\hline MPV & $8.70 \pm 1.10$ & $8.54 \pm 1.06$ & $8.43 \pm 1.41$ & 0.233 & $8.77 \pm 1.11$ & $8.71 \pm 1.11$ & $8.40 \pm 1.08$ & 0.007 \\
\hline РCT & $0.22 \pm 0.07$ & $0.22 \pm 0.08$ & $0.20 \pm 0.06$ & 0.334 & $0.22 \pm 0.07$ & $0.22 \pm 0.07$ & $0.22 \pm 0.07$ & 0.955 \\
\hline L1 & $-3.09 \pm 0.71$ & $-3.13 \pm 0.65$ & $-3.09 \pm 0.36$ & 0.810 & $-2.95 \pm 0.66$ & $-3.09 \pm 0.71$ & $-3.22 \pm 0.63$ & 0.006 \\
\hline L2 & $-3.20 \pm 0.65$ & $-3.22 \pm 0.63$ & $-2.82 \pm 0.60$ & 0.027 & $-3.08 \pm 0.65$ & $-3.21 \pm 0.61$ & $-3.21 \pm 0.69$ & 0.186 \\
\hline L3 & $-3.10 \pm 0.69$ & $-3.10 \pm 0.70$ & $-2.78 \pm 0.51$ & 0.117 & $-3.08 \pm 0.61$ & $-3.09 \pm 0.63$ & $-3.08 \pm 0.80$ & 0.968 \\
\hline L4 & $-3.30 \pm 0.76$ & $-3.27 \pm 0.65$ & $-2.83 \pm 0.37$ & 0.017 & $-3.25 \pm 0.77$ & $-3.30 \pm 0.68$ & $-3.24 \pm 0.76$ & 0.685 \\
\hline \multirow{2}{*}{$\begin{array}{l}\text { Clinical } \\
\text { indicators }\end{array}$} & \multicolumn{3}{|c|}{ WLS-rs7554551 } & \multicolumn{2}{|c|}{ WNT16-rs917727 } & & & \\
\hline & TT & TC & $\mathrm{CC}$ & $\mathrm{P}$ & TT & TC & $\mathrm{CC}$ & $\mathrm{P}$ \\
\hline UA & $\begin{array}{l}262.99 \pm \\
89.07\end{array}$ & $\begin{array}{l}257.37 \pm \\
80.30\end{array}$ & $\begin{array}{l}253.53 \pm \\
64.08\end{array}$ & 0.318 & $\begin{array}{l}254.43 \pm \\
43.18\end{array}$ & $\begin{array}{l}258.35 \pm \\
78.45\end{array}$ & $261.41 \pm 87.59$ & 0.929 \\
\hline Urea & $5.96 \pm 2.04$ & $5.74 \pm 1.99$ & $6.18 \pm 2.31$ & 0.880 & $6.11 \pm 1.96$ & $6.10 \pm 2.41$ & $5.83 \pm 1.92$ & 0.444 \\
\hline Fasting glucose & $4.74 \pm 1.19$ & $4.81 \pm 1.23$ & $4.71 \pm 1.14$ & 0.217 & $5.13 \pm 1.53$ & $4.67 \pm 1.24$ & $4.79 \pm 1.19$ & 0.488 \\
\hline WBC & $7.18 \pm 2.48$ & $7.51 \pm 2.89$ & $7.56 \pm 2.25$ & 0.365 & $6.93 \pm 2.00$ & $7.41 \pm 2.86$ & $7.29 \pm 2.57$ & 0.849 \\
\hline $\mathrm{RBC}$ & $4.35 \pm 0.54$ & $4.28 \pm 0.55$ & $4.35 \pm 0.43$ & 0.381 & $4.17 \pm 0.68$ & $4.27 \pm 0.55$ & $4.34 \pm 0.53$ & 0.336 \\
\hline PLT & $\begin{array}{l}248.62 \pm \\
95.82\end{array}$ & $\begin{array}{l}277.75 \pm \\
113.86\end{array}$ & $\begin{array}{l}252.95 \pm \\
103.82\end{array}$ & 0.010 & $\begin{array}{l}312.57 \pm \\
147.00\end{array}$ & $\begin{array}{l}267.89 \pm \\
97.50\end{array}$ & $\begin{array}{l}255.61 \pm \\
104.11\end{array}$ & 0.212 \\
\hline PDW & $13.02 \pm 3.34$ & $12.20 \pm 3.43$ & $11.80 \pm 2.75$ & 0.017 & $10.57 \pm 2.31$ & $12.54 \pm 3.46$ & $12.77 \pm 3.36$ & 0.203 \\
\hline MPV & $8.74 \pm 1.10$ & $8.45 \pm 1.11$ & $8.36 \pm 1.02$ & 0.011 & $7.87 \pm 0.81$ & $8.56 \pm 1.11$ & $8.66 \pm 1.11$ & 0.128 \\
\hline РCT & $0.21 \pm 0.07$ & $0.23 \pm 0.08$ & $0.21 \pm 0.08$ & 0.045 & $0.24 \pm 0.10$ & $0.22 \pm 0.07$ & $0.22 \pm 0.07$ & 0.460 \\
\hline L1 & $-3.06 \pm 0.69$ & $-3.15 \pm 0.68$ & $-3.21 \pm 0.49$ & 0.256 & $-2.94 \pm 0.16$ & $-3.23 \pm 0.68$ & $-3.07 \pm 0.68$ & 0.076 \\
\hline L2 & $-3.18 \pm 0.65$ & $-3.23 \pm 0.65$ & $-2.91 \pm 0.50$ & 0.100 & $-3.04 \pm 0.28$ & $-3.34 \pm 0.62$ & $-3.15 \pm 0.65$ & 0.015 \\
\hline L3 & $-3.12 \pm 0.67$ & $-3.05 \pm 0.70$ & $-2.84 \pm 0.64$ & 0.164 & $-2.76 \pm 0.36$ & $-3.23 \pm 0.69$ & $-3.05 \pm 0.68$ & 0.023 \\
\hline L4 & $-3.29 \pm 0.76$ & $-3.21 \pm 0.83$ & $-3.21 \pm 0.83$ & 0.745 & $-3.21 \pm 0.40$ & $-3.38 \pm 0.71$ & $-3.24 \pm 0.73$ & 0.176 \\
\hline \multirow{2}{*}{$\begin{array}{l}\text { Clinical } \\
\text { indicators }\end{array}$} & \multicolumn{3}{|c|}{ WNT16-rs7776725 } & \multicolumn{2}{|c|}{ LRP-rs2291467 } & & & \\
\hline & TT & $\mathrm{TC}$ & $\mathrm{CC}$ & $\mathrm{P}$ & TT & CT & $\mathrm{CC}$ & $\mathrm{P}$ \\
\hline UA & $\begin{array}{l}260.71 \pm \\
87.44\end{array}$ & $\begin{array}{l}260.93 \pm \\
79.42\end{array}$ & $\begin{array}{l}254.43 \pm \\
43.18\end{array}$ & 0.981 & $\begin{array}{l}259.00 \pm \\
124.97\end{array}$ & $\begin{array}{l}246.28 \pm \\
77.54\end{array}$ & $268.03 \pm 87.62$ & 0.024 \\
\hline Urea & $5.83 \pm 1.93$ & $6.08 \pm 2.38$ & $6.11 \pm 1.96$ & 0.505 & $6.04 \pm 1.88$ & $5.97 \pm 2.11$ & $5.89 \pm 2.03$ & 0.463 \\
\hline Fasting glucose & $4.79 \pm 1.19$ & $4.67 \pm 1.22$ & $5.13 \pm 1.53$ & 0.468 & $4.97 \pm 1.35$ & $4.69 \pm 0.92$ & $4.80 \pm 1.32$ & 0.540 \\
\hline
\end{tabular}




\begin{tabular}{|c|c|c|c|c|c|c|c|c|}
\hline \multirow{2}{*}{$\begin{array}{l}\text { Clinical } \\
\text { indicators }\end{array}$} & \multicolumn{4}{|c|}{ WLS-rs2566755 } & \multicolumn{4}{|c|}{ WLS-rs2566752 } \\
\hline & TT & CT & $\mathrm{CC}$ & $\mathbf{P}$ & TT & CT & $\mathrm{CC}$ & $\mathbf{P}$ \\
\hline WBC & $7.29 \pm 2.57$ & $7.42 \pm 2.84$ & $6.93 \pm 2.00$ & 0.821 & $6.54 \pm 1.03$ & $7.30 \pm 2.83$ & $7.33 \pm 2.53$ & 0.801 \\
\hline RBC & $4.34 \pm 0.53$ & $4.27 \pm 0.55$ & $4.17 \pm 0.68$ & 0.314 & $3.90 \pm 0.19$ & $4.33 \pm 0.56$ & $4.33 \pm 0.53$ & 0.198 \\
\hline PLT & $\begin{array}{l}255.32 \pm \\
104.09\end{array}$ & $\begin{array}{l}268.48 \pm \\
97.73\end{array}$ & $\begin{array}{l}312.57 \pm \\
147.00\end{array}$ & 0.191 & $\begin{array}{l}329.20 \pm \\
104.24\end{array}$ & $\begin{array}{l}248.40 \pm \\
97.99\end{array}$ & $\begin{array}{l}263.30 \pm \\
105.76\end{array}$ & 0.096 \\
\hline PDW & $12.78 \pm 3.36$ & $12.53 \pm 3.45$ & $10.57 \pm 2.31$ & 0.197 & $10.45 \pm 2.29$ & $12.84 \pm 3.19$ & $12.65 \pm 3.48$ & 0.276 \\
\hline MPV & $8.66 \pm 1.11$ & $8.56 \pm 1.10$ & $7.87 \pm 0.81$ & 0.124 & $7.86 \pm 0.78$ & $8.71 \pm 1.07$ & $8.60 \pm 1.12$ & 0.171 \\
\hline РСт & $0.22 \pm 0.07$ & $0.22 \pm 0.07$ & $0.24 \pm 0.10$ & 0.425 & $0.25 \pm 0.07$ & $0.21 \pm 0.08$ & $0.22 \pm 0.07$ & 0.351 \\
\hline L1 & $-3.07 \pm 0.69$ & $-3.21 \pm 0.68$ & $-2.94 \pm 0.16$ & 0.120 & $-3.44 \pm 0.80$ & $-3.03 \pm 0.57$ & $-3.13 \pm 0.73$ & 0.173 \\
\hline L2 & $-3.15 \pm 0.65$ & $-3.33 \pm 0.62$ & $-3.04 \pm 0.28$ & 0.029 & $-3.58 \pm 0.68$ & $-3.11 \pm 0.65$ & $-3.22 \pm 0.64$ & 0.095 \\
\hline L3 & $-3.05 \pm 0.68$ & $-3.23 \pm 0.71$ & $-2.76 \pm 0.36$ & 0.025 & $-3.70 \pm 0.92$ & $-3.00 \pm 0.66$ & $-3.12 \pm 0.69$ & 0.018 \\
\hline L4 & $-3.24 \pm 0.73$ & $-3.38 \pm 0.70$ & $-3.21 \pm 0.40$ & 0.198 & $-4.00 \pm 1.53$ & $-3.19 \pm 0.67$ & $-3.30 \pm 0.73$ & 0.024 \\
\hline \multirow{2}{*}{$\begin{array}{l}\text { Clinical } \\
\text { indicators }\end{array}$} & \multicolumn{3}{|c|}{ LRP-rs11228240 } & \multicolumn{3}{|c|}{ LRP-rs12272917 } & & \\
\hline & TT & CT & $\mathrm{CC}$ & $\mathrm{P}$ & TT & TC & $\mathrm{CC}$ & $\mathrm{P}$ \\
\hline UA & $\begin{array}{l}259.00 \pm \\
124.97\end{array}$ & $\begin{array}{l}246.31 \pm \\
76.77\end{array}$ & $\begin{array}{l}268.34 \pm \\
88.09\end{array}$ & 0.021 & $\begin{array}{l}267.07 \pm \\
87.64\end{array}$ & $\begin{array}{l}248.04 \pm \\
78.03\end{array}$ & $\begin{array}{l}256.14 \pm \\
109.65\end{array}$ & 0.059 \\
\hline Urea & $6.04 \pm 1.34$ & $5.74 \pm 1.87$ & $5.97 \pm 2.13$ & 0.479 & $5.96 \pm 2.11$ & $5.74 \pm 1.87$ & $6.11 \pm 1.88$ & 0.478 \\
\hline Fasting glucose & $4.97 \pm 1.35$ & $4.69 \pm 0.92$ & $4.80 \pm 1.33$ & 0.564 & $4.81 \pm 1.32$ & $4.68 \pm 0.93$ & $4.74 \pm 1.18$ & 0.539 \\
\hline WBC & $6.54 \pm 1.03$ & $7.32 \pm 2.83$ & $7.32 \pm 2.53$ & 0.805 & $7.29 \pm 2.54$ & $7.39 \pm 2.82$ & $6.34 \pm 1.06$ & 0.566 \\
\hline RBC & $3.90 \pm 0.19$ & $4.33 \pm 0.56$ & $4.33 \pm 0.53$ & 0.199 & $4.34 \pm 0.53$ & $4.31 \pm 0.55$ & $4.11 \pm 0.41$ & 0.496 \\
\hline PLT & $\begin{array}{l}329.20 \pm \\
104.24\end{array}$ & $\begin{array}{l}249.94 \pm \\
101.93\end{array}$ & $\begin{array}{l}262.70 \pm \\
103.98\end{array}$ & 0.130 & $\begin{array}{l}260.45 \pm \\
103.70\end{array}$ & $\begin{array}{l}254.41 \pm \\
103.25\end{array}$ & $\begin{array}{l}296.86 \pm \\
104.19\end{array}$ & 0.514 \\
\hline PDW & $10.45 \pm 2.29$ & $12.76 \pm 3.19$ & $12.69 \pm 3.48$ & 0.322 & $12.67 \pm 3.46$ & $12.79 \pm 3.21$ & $11.50 \pm 3.24$ & 0.595 \\
\hline MPV & $7.86 \pm 0.78$ & $8.68 \pm 1.08$ & $8.61 \pm 1.12$ & 0.237 & $8.62 \pm 1.14$ & $8.67 \pm 1.05$ & $8.25 \pm 1.11$ & 0.591 \\
\hline РСТ & $0.25 \pm 0.07$ & $0.21 \pm 0.08$ & $0.22 \pm 0.07$ & 0.377 & $0.22 \pm 0.07$ & $0.22 \pm 0.08$ & $0.24 \pm 0.06$ & 0.794 \\
\hline L1 & $-3.44 \pm 0.80$ & $-3.04 \pm 0.57$ & $-3.13 \pm 0.73$ & 0.188 & $-3.11 \pm 0.72$ & $-3.06 \pm 0.58$ & $-3.43 \pm 0.77$ & 0.313 \\
\hline L2 & $-3.58 \pm 0.68$ & $-3.10 \pm 0.65$ & $-3.23 \pm 0.64$ & 0.041 & $-3.18 \pm 0.65$ & $-3.18 \pm 0.64$ & $-3.43 \pm 0.79$ & 0.597 \\
\hline L3 & $-3.70 \pm 0.92$ & $-2.98 \pm 0.65$ & $-3.13 \pm 0.69$ & 0.009 & $-3.11 \pm 0.69$ & $-3.02 \pm 0.66$ & $-3.60 \pm 0.84$ & 0.052 \\
\hline L4 & $-4.00 \pm 1.53$ & $-3.19 \pm 0.67$ & $-3.30 \pm 0.73$ & 0.017 & $-3.30 \pm 0.71$ & $-3.18 \pm 0.70$ & $-3.76 \pm 1.37$ & 0.039 \\
\hline
\end{tabular}

MDR analysis

We used MDR analysis to assess the effect of SNP-SNP interactions among the eleven selected SNPs in WLS, WNT16 and LRP5 (Table 6, Supplemental Table 4-6). In total, we found four models, and a four-locus model including rs 2566752 and rs 2566755 in $W L S$, rs 7776725 in $W N T 16$ and rs 12272917 in $\angle R P 5$ was the best model (CV consistency $=9 / 10$, accuracy $=0.605, P<0.001)$. We then showed SNP-SNP interactions with genotype combinations (Fig. 4-6) and revealed that the impact of the SNP genotypes of three genes on osteoporosis risk are dependent on one another. 
Table 6

MDR analysis of SNP-SNP interactions

\begin{tabular}{|c|c|c|c|c|c|c|c|c|}
\hline Model & $\begin{array}{l}\text { Training } \\
\text { Bal. Acc. }\end{array}$ & $\begin{array}{l}\text { Testing } \\
\text { Bal. Acc. }\end{array}$ & $\begin{array}{l}\text { CV } \\
\text { Consistency }\end{array}$ & Accuracy & Sensitivity & Specificity & OR(95\%Cl) & $P$ \\
\hline WLS rs2566755 & 0.528 & 0.488 & $5 / 10$ & 0.525 & 0.669 & 0.380 & $\begin{array}{l}1.24(0.96- \\
1.60)\end{array}$ & 0.102 \\
\hline WLS rs2566755, LRP5 rs12272917 & 0.550 & 0.497 & $5 / 10$ & 0.546 & 0.810 & 0.282 & $\begin{array}{l}1.67(1.25- \\
2.25)\end{array}$ & 0.001 \\
\hline $\begin{array}{l}\text { WLS rs2566752, WLS rs2566755, } \\
\text { WNT16 rs3801387 }\end{array}$ & 0.581 & 0.549 & $6 / 10$ & 0.580 & 0.561 & 0.597 & $\begin{array}{l}1.91(1.49- \\
2.45)\end{array}$ & ¿. 001 \\
\hline $\begin{array}{l}\text { WLS rs2566752, WLS rs2566755, } \\
\text { WNT16 rs7776725, LRP5 } \\
\text { rs } 12270917\end{array}$ & 0.607 & 0.563 & $9 / 10$ & 0.605 & 0.732 & 0.478 & $\begin{array}{l}2.49(1.92- \\
3.24)\end{array}$ & $<.001$ \\
\hline
\end{tabular}

MDR, multifactor dimensionality reduction; SNP, single nucleotide polymorphism; CV, cross-validation; OR, odds ratio; $\mathrm{Cl}$, confidence interval.

Bold values mean statistical significance.

\section{Discussion}

In this study, we investigated the association between candidate genetic variations (WLS. rs2566755, rs12407028, rs2566752 and rs7554551; WNT16: rs3779381, rs3801387, rs917727 and rs7776725; LRP5: rs2291467, rs11228240 and rs12272917) of the WNT signaling pathway and osteoporosis risk among Chinese postmenopausal women. We found a strong relationship between LRP5 polymorphisms (rs2291467, rs11228240 and rs12272917) and osteoporosis risk, and this effect was BMI-dependent. In the subgroup of BMI > 24, WNT16 polymorphisms (rs3779381, rs3801387, rs917727 and rs7776725) were significantly associated with an increased risk of osteoporosis $(P<0.05)$. Haplotype analysis showed that three blocks (block 1: rs2566755 and rs12407028, block 2: rs3779381, rs3801387, rs917727 and rs7776725 and block 3: rs2291467 and rs11228240). Additionally, MDR analysis revealed that a four-locus SNP-SNP interaction pattern (rs2566752 in WLS, rs2566755 in WLS, rs7776725 in WNT16 and rs12272917 in LRP5) was significantly associated with osteoporosis risk.

Osteoporosis is a disease in which bone density and strength decrease, leading to skeletal dysfunction [32]. The WNT signaling pathway is one of the major signaling pathways involved in maintaining bone homeostasis and is also a key signaling pathway of other biological processes, such as embryogenesis, organogenesis and tumorigenesis[33]. It is reported that reduced WNT signaling results in increased osteoclast activity and decreased osteoblast activity[5]. Several loci clustered within the WNT signaling pathway are strongly associated with BMD, including WLS, WNT16 and LRP5. WLS is an integral component of the WNT ligand secretion pathway, which is required for the development of various organs[34]. Genetic variation in the WLS gene has been studied in several GWAS; rs2566752 is the maximally associated variant for lumbar spine and femoral neck BMD[35,36]. However, the association of WLS polymorphisms and the risk of BMD or osteoporosis is inconsistent[37], which may be attributed to a limited sample size and ethnicity differences. In our study, we did not observe a strong relationship between WLS polymorphisms (rs2566755, rs12407028, rs2566752 and rs7554551) and osteoporosis susceptibility among Chinese postmenopausal women. Nevertheless, we found a significant association between some clinical indicators and different genotypes of WLSpolymorphisms, suggesting that genotypes of WLS polymorphisms have effects on the level of clinical factors.

WNT16 is a noncanonical WNT ligand that regulates bone resorption and thus inhibits osteoporosis[38]. It is reported that genetic polymorphisms of WNT16 are associated with BMD at different bone sites. Although the relationship of rs3801387 and BMD is controversial[39], we found that rs3801387 significantly increased the osteoporosis risk of Chinese postmenopausal women with higher BMI (>24), suggesting the potential importance of this variant in osteoporosis. Rs917727 is another WNT16 polymorphism; a GWAS showed that rs917727 influences skull BMD[25]. Consistent with previous studies, we observed a strong linkage of rs917727 and osteoporosis risk in stratification analysis. In addition, we assessed the relationship of two other polymorphisms (rs3779381 and rs7776725) with osteoporosis. We first found that individuals carrying the heterozygote genotype of rs3779381 had higher osteoporosis risk. In Caucasians, rs7776725 is associated with hip, spine, wrist and whole-body BMD[26], whereas rs7776725 is associated with osteoporosis in Chinese women with higher BMI. These studies revealed that WNT16 polymorphisms (rs3779381, rs3801387, rs917727 and rs7776725) might affect the risk of osteoporosis. Further studies are needed to confirm the role of WNT16 polymorphisms on the pathology of osteoporosis.

LRP5 encodes a transmembrane protein in the WNT signaling pathway and regulates bone mass in humans and mice[40]. Several studies have shown that $L R P 5$ polymorphisms are a determining factor of bone mass[41]. In this study, we first found that rs2291467, rs 11228240 and rs12272917 of $\angle R P 5$ were significantly associated with a decreased risk of osteoporosis and that the relationships were BMI-dependent. We also observed the association of two LRP5 haplotypes ( $\mathrm{T}_{\mathrm{rs} 2291467} \mathrm{~T}_{\mathrm{rs} 11228240}$ and $\left.\mathrm{C}_{\mathrm{rs} 2291467} \mathrm{C}_{\mathrm{rs} 11228240}\right)$ with osteoporosis in Chinese postmenopausal women. These results provided further insight into the cause of osteoporosis; however, the complete mechanism of osteoporosis requires further 
exploration. Moreover, MDR analysis elucidated the association between gene-gene interactions and osteoporosis risk in the Chinese population. Additional studies to determine the exact mechanism of gene-gene interactions on osteoporosis treatment are necessary.

Some limitations of this study are noteworthy. First, we did not have information about lifestyle habits, physical activity or other clinical indicators, and therefore, we could not explore the associations with these variables or adjust them. Second, we were not able to genotype all the polymorphisms in WLS, WNT16 and LRP5. Third, all the participants in this study were enrolled from the same hospital, and therefore, selection biases are not neglected. Furthermore, functional experiments should be conducted in a larger sample to confirm the relationship of WNT signaling pathway related genes and osteoporosis risk.

\section{Conclusions}

In summary, we found a significant association of WNT signaling pathway related genes (WLS, WNT16 and LRP5) and osteoporosis risk among Chinese postmenopausal women. More individuals from different areas and countries in further studies are needed to verify our findings.

\section{Abbreviations List}

Multifactor Dimensionality Reduction (MDR)

Bone mineral density (BMD)

Wntless (WLS)

Wnt family member $16($ WNT16)

Genome-wide association studies (GWAS)

Low-density lipoprotein receptor-related protein 5 (LRP5)

Linkage disequilibrium (LD)

Hardy-Weinberg equilibrium (HWE)

Body mass index (BMI)

Uric acid (UA)

White blood cell (WBC)

Red blood cell (RBC)

Platelets (PLT)

Platelet distribution width (PDW)

Mean platelet volume (MPV)

Plateletocrit (PCT)

\section{Declarations}

\section{Funding}

Not applicable.

\section{Acknowledgement}

We sincerely thank all the participants in this study.

\section{Competing interests}

We declare that there were no conflicts of interest.

Data Availability 
All relevant data are within the manuscript.

\section{Ethical Approval and Consent to participate}

This study was performed in accordance with the World Medical Association Declaration of Helsinki and was approved by the Ethics Committee of Xi'an 630 Hospital. Written informed consent was obtained from all of the subjects before participating.

\section{Consent for publication}

Not applicable

\section{Authors' contributions}

ZY and JL completed genotyping and performed the manuscript. JJ F, ZJ C and SW L participated in the statistical analysis of the data and modified the manuscript. YS designed the study, co-supervised the work and modified the manuscript. All the authors have read and approved the final manuscript.

\section{Authors' information}

${ }^{1}$ Beijing Institute of Brain Disorders, Laboratory of Brain Disorders, Ministry of Science and Technology, Collaborative Innovation Center for Brain Disorders, Capital Medical University, 100069, Beijing, China. ${ }^{2}$ Department of Internal Neurology, Inner Mongolia Medical University Affiliated Hospital, 10050. ${ }^{3}$ Department of Minimal Invasive Spine Surgery, The Second Affiliated Hospital of Inner Mongolia Medical College, 10000.

\section{References}

1. Gao S-t, Lv Z-t, Zhou C-k, Mao C. Sheng W-b: Association between IGF-1 polymorphisms and risk of osteoporosis in Chinese population: a meta-analysis. BMC Musculoskelet Disord. 2018;19(1):141

2. Sugimoto T, Sato M, Dehle FC, Brnabic AJ, Weston A, Burge R. Lifestyle-related metabolic disorders, osteoporosis, and fracture risk in Asia: A systematic review. Value in health regional issues. 2016;9:49-56.

3. Al-Daghri NM, Yakout S, Al-Shehri E, Al-Fawaz H, Aljohani N, Al-Saleh Y. Inflammatory and bone turnover markers in relation to PTH and vitamin D status among Saudi postmenopausal women with and without osteoporosis. Int J Clin Exp Med. 2014;7(9):2812.

4. Wang Y, Tao Y, Hyman M, Li J, Chen Y. Osteoporosis in china. Osteoporosis international. 2009;20(10):1651-62.

5. Zhang D, Ge Z, Ma X, Zhi L, Zhang Y, Wu X, Yao S, Ma W. Genetic association study identified a 20 kb regulatory element in WLS associated with osteoporosis and bone mineral density in Han Chinese. Scientific reports. 2017;7(1):13668.

6. Lim WH, Liu B, Hunter DJ, Cheng D, Mah S-j, Helms JA. Downregulation of Wnt causes root resorption. Am J Orthod Dentofac Orthop. 2014;146(3):337-45.

7. Horváth P, Balla B, Kósa JP, Tóbiás B, Szili B, Kirschner G, Győri G, Kató K, Lakatos P, Takács I. Strong effect of SNP rs4988300 of the LRP5 gene on bone phenotype of Caucasian postmenopausal women. J Bone Miner Metab. 2016;34(1):79-85.

8. Todd H, Galea GL, Meakin LB, Delisser PJ, Lanyon LE, Windahl SH, Price JS. Wnt16 is associated with age-related bone loss and estrogen withdrawal in murine bone. PLoS One. 2015;10(10):e0140260.

9. Yang PT, Anastas JN, Toroni RA, Shinohara MM, Goodson JM, Bosserhoff AK, Chien AJ, Moon RT. WLS inhibits melanoma cell proliferation through the $\beta$-catenin signalling pathway and induces spontaneous metastasis. EMBO molecular medicine. 2012;4(12):1294-307.

10. Harterink M, Port F, Lorenowicz MJ, McGough IJ, Silhankova M, Betist MC, Van Weering JR, Van Heesbeen RG, Middelkoop TC, Basler K. A SNX3-dependent retromer pathway mediates retrograde transport of the Wnt sorting receptor Wntless and is required for Wnt secretion. Nat Cell Biol. 2011;13(8):914.

11. Silhankova M, Port F, Harterink M, Basler K, Korswagen HC. Wnt signalling requires MTM-6 and MTM-9 myotubularin lipid-phosphatase function in Wnt-producing cells. EMBO J. 2010;29(24):4094-105.

12. Yang P-T, Lorenowicz MJ, Silhankova M, Coudreuse DY, Betist MC, Korswagen HC. Wnt signaling requires retromer-dependent recycling of MIG-14/Wntless in Wnt-producing cells. Developmental cell. 2008;14(1):140-7.

13. Coombs GS, Yu J, Canning CA, Veltri CA, Covey TM, Cheong JK, Utomo V, Banerjee N, Zhang ZH, Jadulco RC. WLS-dependent secretion of WNT3A requires Ser209 acylation and vacuolar acidification. J Cell Sci. 2010;123(19):3357-67.

14. Fu J, Jiang M, Mirando AJ, Yu H-MI, Hsu W: Reciprocal regulation of Wnt and Gpr177/mouse Wntless is required for embryonic axis formation. Proceedings of the National Academy of Sciences 2009, 106(44):18598-18603.

15. Bafico A, Liu G, Goldin L, Harris V, Aaronson SA. An autocrine mechanism for constitutive Wnt pathway activation in human cancer cells. Cancer cell. 2004;6(5):497-506. 
16. Rask K, Nilsson A, Brännström M, Carlsson P, Hellberg P, Janson P, Hedin L, Sundfeldt K. Wnt-signalling pathway in ovarian epithelial tumours: increased expression of $\beta$-catenin and GSK3ß. British journal of cancer. 2003;89(7):1298.

17. Badiglian Filho L, Oshima CTF, De Oliveira Lima F, De Oliveira Costa H, De Sousa Damião R, Gomes TS, Goncalves WJ. Canonical and noncanonical Wnt pathway: a comparison among normal ovary, benign ovarian tumor and ovarian cancer. Oncol Rep. 2009;21(2):313-20.

18. Pan K-F, Liu W-G, Zhang L, You W-C, Lu Y-Y. Mutations in components of the Wnt signaling pathway in gastric cancer. World journal of gastroenterology: WJG. 2008;14(10):1570.

19. Maruyama T, Jiang M, Hsu W. Gpr177, a novel locus for bone mineral density and osteoporosis, regulates osteogenesis and chondrogenesis in skeletal development. J Bone Miner Res. 2013;28(5):1150-9.

20. Schrier SA, Bodurtha JN, Burton B, Chudley AE, Chiong MAD, D'avanzo MG, Lynch SA, Musio A, Nyazov DM, Sanchez-Lara PA. The Coffin-Siris syndrome: A proposed diagnostic approach and assessment of 15 overlapping cases. American Journal of Medical Genetics Part A. 2012;158(8):1865-76.

21. Fear MW, Kelsell DP, Spurr NK, Barnes MR. Wnt-16a, a novel Wnt-16 isoform, which shows differential expression in adult human tissues. Biochem Biophys Res Commun. 2000;278(3):814-20.

22. Gori F, Lerner U, Ohlsson C, Baron R: A new WNT on the bone: WNT16, cortical bone thickness, porosity and fractures. BoneKEy reports $2015,4$.

23. Wergedal JE, Kesavan C, Brommage R, Das S, Mohan S. Role of WNT16 in the regulation of periosteal bone formation in female mice. Endocrinology. 2015;156(3):1023-32.

24. Koller DL, Zheng HF, Karasik D, Yerges-Armstrong L, Liu CT, McGuigan F, Kemp JP, Giroux S, Lai D, Edenberg HJ. Meta-analysis of genome-wide studies identifies WNT16 and ESR1 SNPs associated with bone mineral density in premenopausal women. J Bone Miner Res. 2013;28(3):547-58.

25. Medina-Gomez C, Kemp JP, Estrada K, Eriksson J, Liu J, Reppe S, Evans DM, Heppe DH, Vandenput L, Herrera L. Meta-analysis of genome-wide scans for total body BMD in children and adults reveals allelic heterogeneity and age-specific effects at the WNT16 locus. PLoS Genet. 2012;8(7):e1002718.

26. Zhang L-S, Hu H-G, Liu Y-J, Li J, Yu P, Zhang F, Yang T-L, Tian Q, Zheng Y-P, Guo Y. A follow-up association study of two genetic variants for bone mineral density variation in Caucasians. Osteoporos Int. 2012;23(7):1867-75.

27. Garcia-Ibarbia C, Perez-Nunez M, Olmos J, Valero C, Perez-Aguilar M, Hernandez J, Zarrabeitia M, Gonzalez-Macias J, Riancho J. Missense polymorphisms of the WNT16 gene are associated with bone mass, hip geometry and fractures. Osteoporos Int. 2013;24(9):2449-54.

28. Gong Y, Slee RB, Fukai N, Rawadi G, Roman-Roman S, Reginato AM, Wang H, Cundy T, Glorieux FH, Lev D. LDL receptor-related protein 5 (LRP5) affects bone accrual and eye development. Cell. 2001;107(4):513-23.

29. Mammoto A, Muyleart M, Mammoto T. LRP5 in age-related changes in vascular and alveolar morphogenesis in the lung. Aging. $2019 ; 11(1): 89$.

30. Pepe J, Bonnet N, Herrmann F, Biver E, Rizzoli R, Chevalley T, Ferrari SL. Interaction between LRP5 and periostin gene polymorphisms on serum periostin levels and cortical bone microstructure. Osteoporos Int. 2018;29(2):339-46.

31. Kato M, Patel MS, Levasseur R, Lobov I, Chang BH-J, Glass DA, Hartmann C, Li L, Hwang T-H, Brayton CF. Cbfa1-independent decrease in osteoblast proliferation, osteopenia, and persistent embryonic eye vascularization in mice deficient in Lrp5, a Wnt coreceptor. J Cell Biol. 2002;157(2):303-14.

32. Baron R, Kneissel M. WNT signaling in bone homeostasis and disease: from human mutations to treatments. Nature medicine. 2013;19(2):179.

33. Guañabens N, Gifre L, Peris P. The role of Wnt signaling and sclerostin in the pathogenesis of glucocorticoid-induced osteoporosis. Curr Osteoporos Rep. 2014;12(1):90-7.

34. Yu HMI, Jin Y, Fu J, Hsu W. Expression of Gpr177, a Wnt trafficking regulator, in mouse embryogenesis. Dev Dyn. 2010;239(7):2102-9.

35. Zhang L, Choi HJ, Estrada K, Leo PJ, Li J, Pei Y-F, Zhang Y, Lin Y, Shen H, Liu Y-Z. Multistage genome-wide association meta-analyses identified two new loci for bone mineral density. Human molecular genetics. 2013;23(7):1923-33.

36. Zheng HF, Forgetta V, Hsu YH, Estrada K, Rosello-Diez A, Leo PJ, Dahia CL, Park-Min KH, Tobias JH, Kooperberg C. Whole-genome sequencing identifies EN1 as a determinant of bone density and fracture. Nature. 2015;526(7571):112.

37. Mullin BH, Walsh JP, Zheng H-F, Brown SJ, Surdulescu GL, Curtis C, Breen G, Dudbridge F, Richards JB, Spector TD. Genome-wide association study using family-based cohorts identifies the WLS and CCDC170/ESR1 loci as associated with bone mineral density. BMC Genomics. 2016;17(1):136.

38. Kobayashi Y, Uehara S, Koide M, Takahashi N. The regulation of osteoclast differentiation by Wnt signals. BoneKEy reports $2015,4$.

39. Correa-Rodríguez M, Rio-Valle JS, Rueda-Medina B. Polymorphisms of the WNT16 gene are associated with the heel ultrasound parameter in young adults. Osteoporos Int. 2016;27(3):1057-61.

40. Peng J, Lu Y, Liu Y, Zhan Y. Association of LRP5 Ala1330Val polymorphism with fracture risk: a meta-analysis. Genet Mol Res 2016, 15(1).

41. Falcón-Ramírez E, Casas-Avila L, Cerda-Flores RM, Castro-Hernández C, Rubio-Lightbourn J, Velázquez-Cruz R, Diez-g P, Penaloza-Espinosa R, Valdés-Flores M. Association of LRP5 haplotypes with osteoporosis in Mexican women. Molecular biology reports. 2013;40(3):2705-10.

Page 16/20 
Figures

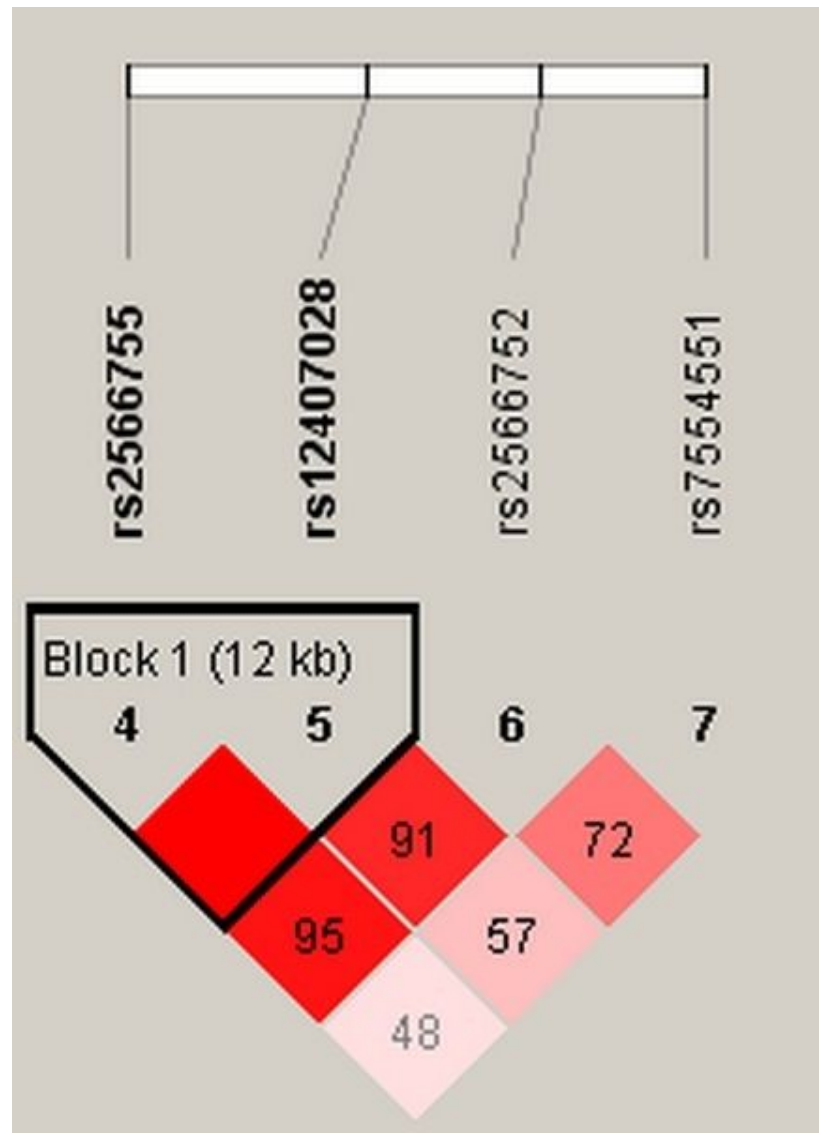

Figure 1

Linkage disequilibrium (LD) plots containing four WLS polymorphisms. Block 1 includes rs2566755 and rs 12407028 . The numbers inside the diamonds indicate the D' for pairwise analyses. 


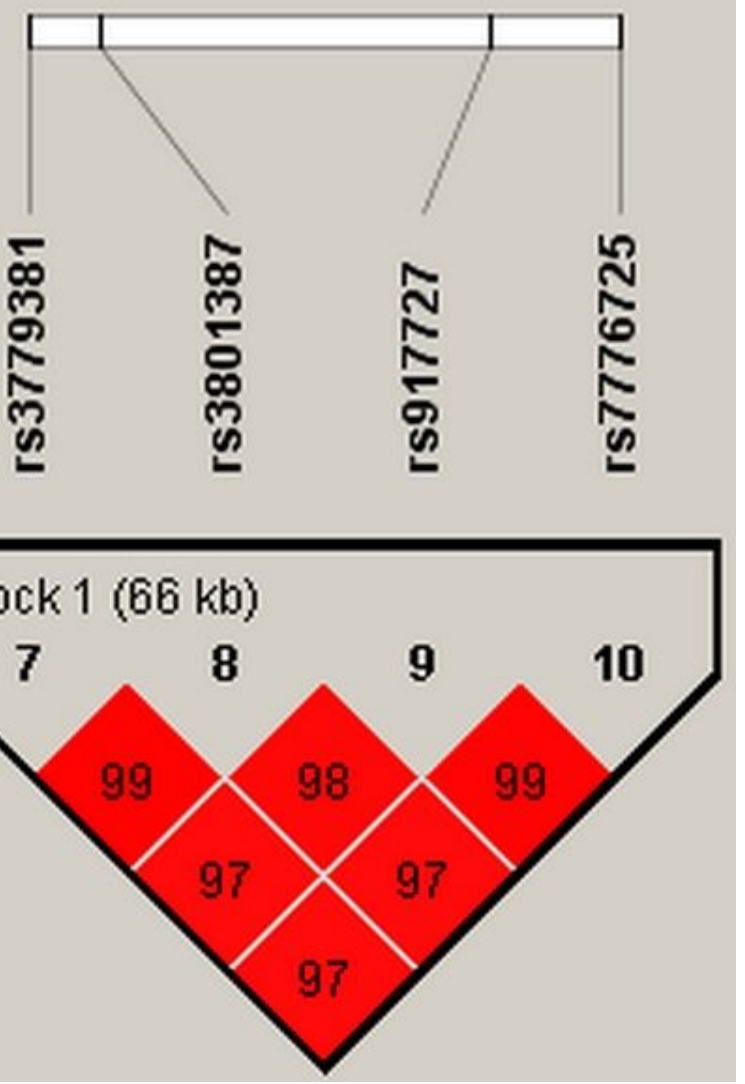

\section{Figure 2}

Linkage disequilibrium (LD) plots containing four WNT16polymorphisms. Block 1 includes rs3779381, rs3801387, rs917727 and rs7776725. The numbers inside the diamonds indicate the $\mathrm{D}^{\prime}$ for pairwise analyses.
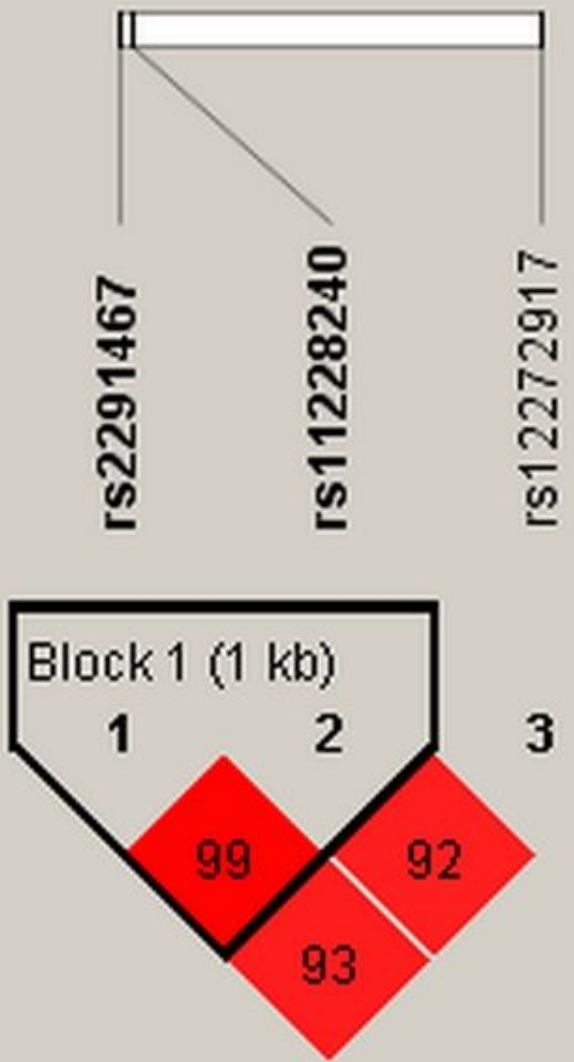
Figure 3

Linkage disequilibrium (LD) plots containing three LRP5polymorphisms. Block 1 includes rs2291467 and rs 11228240. The numbers inside the diamonds indicate the $\mathrm{D} \otimes$ for pairwise analyses.

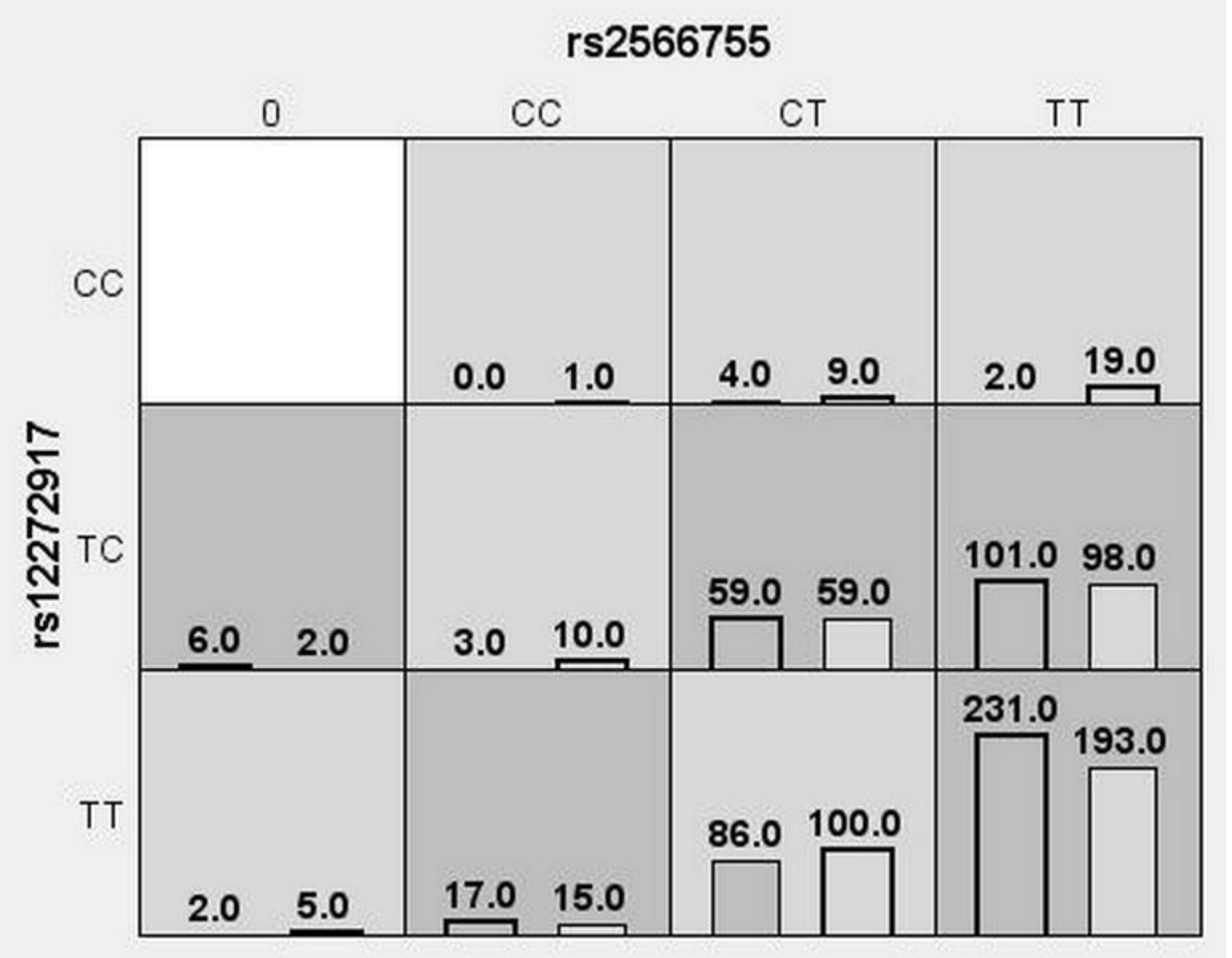

Figure 4

Multifactor dimensionality reduction (MDR) analysis of the WLS rs2566755-LRP5 rs12272917 interaction. In each box, the left bar represents cases and the right bar represents controls. The light gray boxes indicate a low risk of osteoporosis, and the dark gray boxes indicate a high risk of osteoporosis; empty boxes indicate no data.

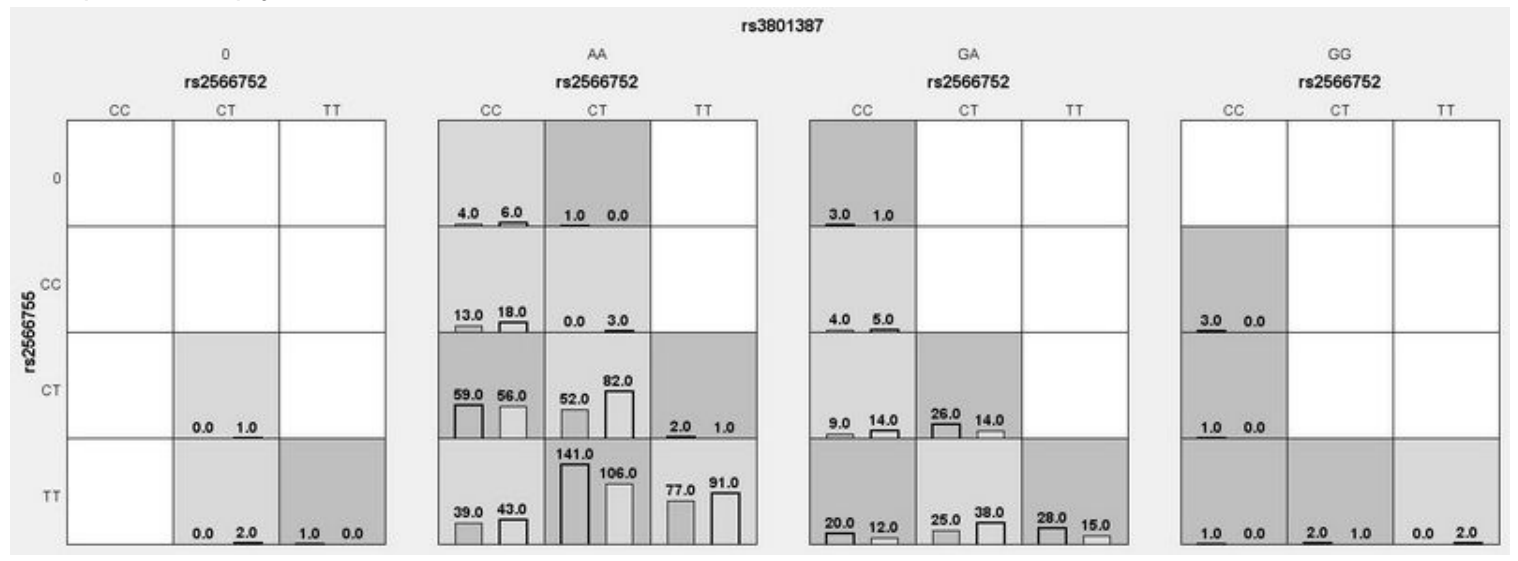

Figure 5

Multifactor dimensionality reduction (MDR) analysis of the WLS rs2566752-WLS rs2566755-WNT16 rs3801387 interaction. In each box, the left bar represents cases and the right bar represents controls. The light gray boxes indicate a low risk of osteoporosis, and the dark gray boxes indicate a high risk of osteoporosis; empty boxes indicate no data. 

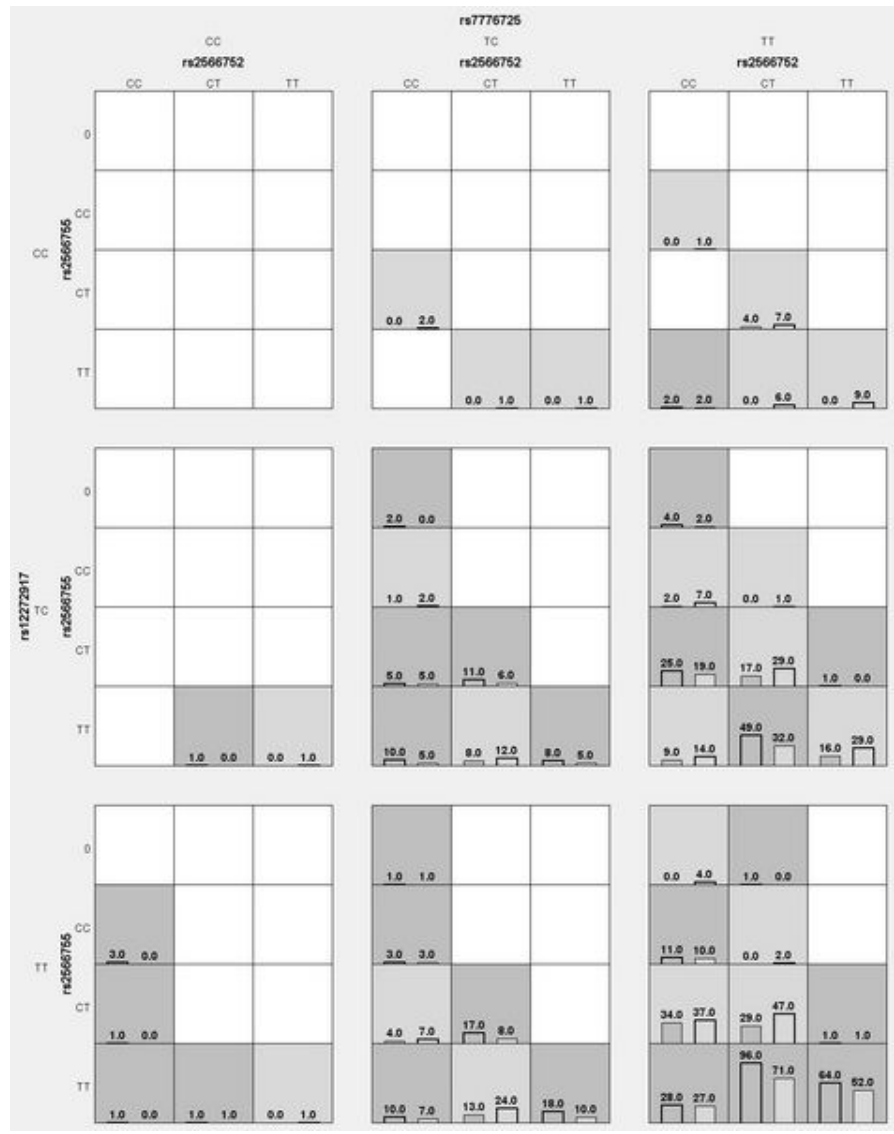

\section{Figure 6}

Multifactor dimensionality reduction (MDR) analysis of the WLS rs2566752-WLS rs2566755-WNT16 rs7776725-LRP5 rs12272917 interaction. In each box, the left bar represents cases and the right bar represents controls. The light gray boxes indicate a low risk of osteoporosis, and the dark gray boxes indicate a high risk of osteoporosis; empty boxes indicate no data.

\section{Supplementary Files}

This is a list of supplementary files associated with this preprint. Click to download.

- SupplementalTables.docx 